\title{
UNDERSTANDING COMPLEX DYNAMICS BY MEANS OF AN ASSOCIATED RIEMANN SURFACE
}

\author{
DAVID GÓMEZ-ULLATE, PAOLO MARIA SANTINI, MATTEO SOMMACAL, AND FRANCESCO CALOGERO
}

\begin{abstract}
We provide an example of how the complex dynamics of a recently introduced model can be understood via a detailed analysis of its associated Riemann surface. Thanks to this geometric description an explicit formula for the period of the orbits can be derived, which is shown to depend on the initial data and the continued fraction expansion of a simple ratio of the coupling constants of the problem. For rational values of this ratio and generic values of the initial data, all orbits are periodic and the system is isochronous. For irrational values of the ratio, there exist periodic and quasi-periodic orbits for different initial data. Moreover, the dependence of the period on the initial data shows a rich behavior and initial data can always be found such the period is arbitrarily high.
\end{abstract}

\section{INTRODUCTION}

Although the evolution of physical systems takes place over real time and can be described by real variables, the interest of extending the study to complex values has long been noticed. For instance, the solutions of a nonlinear system of ODEs representing a physical phenomenon might have singularities off the real time axis whose position depends on the initial data. Even though the time variable is followed along the real axis, the position of these complex singularities provide information on the evolution of the system and its dependence on the choice of initial data.

Historically, one of the first successes of this paradigm is the collection of techniques now known as Painlevé analysis, originally introduced by Painlevé and Kowalevskaya 1, 2. In essence, they consider an ansatz of the local behaviour of a solution near a singularity in terms of a Laurent series, introducing it in the equations and determining the leading orders and resonances (terms in the expansion at which arbitrary constants appear). Painlevé analysis has been extended to test for the presence of algebraic branching (weak Painlevé property 3]). These analytic techniques (which have been algorithmized and are now available in computer packages 4]) constitute a useful tool in the investigation of integrability: in many nonlinear systems where no solution in closed form is known, Painlevé analysis provides information on the type of branching featured by the general solution or by special classes of solutions. It has also proved useful to identify special values of the parameters for which generally chaotic systems such as Hénon-Heiles or Lorenz are integrable [5, 6]. The idea that obstruction to integrability is encoded in the branching properties of solutions and, more precisely, that dense branching is responsible for non integrability, preventing from extracting any useful information on existing first integrals, was first put forward by Kruskal in connection with his poly-Painlevé test [7, 8], a generalization of the Painlevé test allowing one to investigate, in particular, the degree of multivaluedness of the solutions.

Tabor and his collaborators initiated the study of chaotic systems from the point of view of the singularity structure od their solutions 6, 9, 10, 11, 12. Their local analytic approach ( $\Psi$-series) was complemented by numerical techniques developed for finding the location of the singularities in complex time and determining the order of branching [13. Similar studies relating singularity structure, chaos and integrability have been performed by Bountis and his collaborators. They recognised that local analysis alone would not be able to characterise global asymptotic properties of the systems. In a number of papers [14, 15] they propose to call integrable those cases in which the Riemann surface has a finite number of sheets, and non-integrable if the number is infinite. Using mostly numerical evidence they conjecture that in the non-integrable cases the Riemann surfaces are infinitely-sheeted and the projection on the complex plane of the singularities is dense. Combining analytical and numerical results for a simple ODE, Bountis and Fokas [16] have identified chaotic systems with the property that the singularities of their solutions are dense. More recently, a research program to investigate classical dynamics extended to the complex domain is being carried by Bender and

Date: April 13, 2011.

2000 Mathematics Subject Classification. 37Fxx; 37J35; 14H70; 30Fxx.

Key words and phrases. dynamical systems; integrable systems; isochronous systems; Riemann surfaces. 
his collaborators, [17, 18, 19, 20. In these works extensive use is made of numerical explorations to describe complex and interesting behaviours, which include chaotic systems [21, but the necessity to study the Riemann surfaces is recognised.

A precise correspondence between dynamical properties of the system (e.g. chaotic behaviour or sensitive dependence) and specific geometrical properties of the Riemann surface is still an open problem, and this paper can be seen as one more step in this direction. Our motivation was to introduce a model which is simple enough that a full description of its Riemann surface can be performed, yet complicated enough to feature a rich complex behaviour, whose description would not be feasible by standard techniques of qualitative theory. Such a model was initially presented in [22], and in a subsequent paper [23] some results were announced without proof since they required a full description of the Riemann surface, a task which is carried out in this paper. We stress that achieving the complete description of the Riemann surface and its consequence for the determination of the period of the solutions involves the use of various mathematical techniques ranging from complex analysis to combinatorics and showing remarkable connections with number theory (continued fraction expansions).

An important element of the construction of the model is a change of dependent and independent variables which becomes useful to identify isochronous systems [24, 25, 26. Using local analysis and numerical integration in two many-body systems in the plane [27, 28, 29, it was discovered that outside the isochrony region there exist periodic solutions with much higher periods [30, as well as possibly aperiodic solutions, and the connection among this phenomenology and the analytic structure of the corresponding solutions as functions of complex time was illuminated. The model studied in this paper was introduced as a prototype to understand the complex behaviour with many periodic orbits observed numerically in the models described above.

Other attempts to study models which are able to produce chaotic motion and yet lend themselves to a complete description of their Riemann surfaces are those related to the inversion of hyperelliptic integrals [31, 32.

This paper is organized as follows: in Section 2 we present the model, show that it can be reduced to quadratures and how the general solution can be written in terms of a multi-valued function that motivates the study of an associated Riemann surface. In Section 3 we describe the geometric strucure of the Riemann surface, originally for the algebraic case $(\mu \in \mathbb{Q})$. The connection with graph theory and Ferrer diagrams is illustrated in Section 4, which shows also how some results can be framed within the theory of continued fractions. The formulas that allow the explicit determination of the period are given in Section 5, both for the rational and irrational cases. Finally, some conclusions and further work are outlined in Section 6 .

\section{THE MODEL}

The model we analyze in this paper is given by the following set of three coupled first order ODEs:

$$
\dot{z}_{n}+i \omega z_{n}=\frac{g_{n+2}}{z_{n}-z_{n+1}}+\frac{g_{n+1}}{z_{n}-z_{n+2}} .
$$

Notation: Here and hereafter indices such as $n, m$ range from 1 to 3 and are defined Mõd (3), where MõD is defined for all positive integers $a$ and $b$ as follows

$$
a \mathrm{MO} \mathrm{D}(b)=\left\{\begin{array}{cl}
a \mathrm{MOD}(b) & \text { if } a \mathrm{MOD}(b) \neq 0 \\
b & \text { if } a \operatorname{MOD}(b)=0
\end{array} .\right.
$$

(hence, for instance, 3 MÕD $(3)=3,4$ MÕD $(3)=1$, the usefulness of this notation will become apparent below). The dependent variables $z_{n}=z_{n}(t)$ are complex functions and indicate the positions of three interacting bodies in the plane; the independent variable $t$ ("physical time") is real and the superimposed dots denote differentiation with respect to $t$. The parameter $\omega$ is strictly positive and is associated to the period

$$
T=\frac{\pi}{\omega} .
$$

We fix hereafter $\omega=\pi$ without loss of generality so that the fundamental period $T=1$.

The three quantities $g_{n}$ are arbitrary "coupling constants" (possibly also complex; but in this paper we restrict consideration only to the case with real coupling constants). 
In the following we will focus only on the "semisymmetrical case" characterized by the equality of two of the three coupling constants, say

$$
g_{1}=g_{2}=g, \quad g_{3}=f,
$$

since in this case the treatment is simpler yet still adequate to exhibit most aspects of the phenomenology we are interested in (see 23]). In this semisymmetrical case it is convenient to introduce the constant $\mu$,

$$
\mu=\frac{f+2 g}{f+8 g}
$$

whose value, as we shall see, plays an important role in determining the dynamical evolution of our model.

In 23] the general solution of system (11) was given. In the semisymmetrical case (44), if $\mu \neq 0$ and $\mu \neq 1$, the solution of (1) reads as follows.

$$
\begin{gathered}
z_{s}(t)=Z \mathrm{e}^{i \pi t}-\frac{2 z_{3}(0)-z_{1}(0)-z_{2}(0)}{6 \sqrt{\mu}}[\eta \exp (-2 i \pi t)+1]^{1 / 2} . \\
\cdot\left(-[\check{w}(t)]^{1 / 2}+(-1)^{s}[12 \mu-3 \check{w}(t)]^{1 / 2}\right), \quad s=1,2, \\
z_{3}(t)=Z \mathrm{e}^{i \pi t}-\frac{2 z_{3}(0)-z_{1}(0)-z_{2}(0)}{3 \sqrt{\mu}}[\eta \exp (-2 i \pi t)+1]^{1 / 2}[\check{w}(t)]^{1 / 2},
\end{gathered}
$$

where

$$
Z=\frac{z_{1}+z_{2}+z_{3}}{3}
$$

is the center of mass, $\check{w}(t)$ is the solution of the nondifferential equation

$$
[\check{w}(t)-1]^{\mu-1}[\check{w}(t)]^{-\mu}=R \exp (2 i \pi t)+\bar{\xi}=R[\exp (2 i \pi t)+\eta],
$$

and the constants $R, \bar{\xi}$ and $\eta$ are defined in terms of the initial data:

$$
\begin{aligned}
\eta & =\frac{i \pi\left\{\left[z_{1}(0)-z_{2}(0)\right]^{2}+\left[z_{2}(0)-z_{3}(0)\right]^{2}+\left[z_{3}(0)-z_{1}(0)\right]^{2}\right\}}{3(f+2 g)}-1, \\
R & =\frac{3(f+8 g)}{2 i \pi\left[2 z_{3}(0)-z_{1}(0)-z_{2}(0)\right]^{2}}[1-\kappa]^{\mu-1}, \\
\bar{\xi} & =R \eta,
\end{aligned}
$$

where $\kappa$ in (9b) is given by

$$
\kappa=\frac{2 \mu\left[2 z_{3}(0)-z_{1}(0)-z_{2}(0)\right]^{2}}{\left[z_{1}(0)-z_{2}(0)\right]^{2}+\left[z_{2}(0)-z_{3}(0)\right]^{2}+\left[z_{3}(0)-z_{1}(0)\right]^{2}} .
$$

At this stage, it is mandatory to note that (9b) contains a degeneracy of order $\mu-1$. In the following sections we will explain the role of such a degeneracy in the construction of the solution and how to remove it.

If we now set

$$
\begin{aligned}
& \check{w}(t) \equiv w[\xi(t)], \\
& \xi=R \exp (2 i \pi t)+\bar{\xi}=R[\exp (2 i \pi t)+\eta],
\end{aligned}
$$

we can rephrase (8) as follows

$$
[w(\xi)-1]^{\mu-1}[w(\xi)]^{-\mu}=\xi .
$$

Note that this equation is independent of the initial data; it only features the constant $\mu$, which only depends on the coupling constants, see (5). Moreover, it defines the Riemann surface $\Gamma$ consisting of points $(\xi, w) \in \Gamma$ such that (11) is satisfied.

As $\xi$ travels in the complex $\xi$-plane on the circle $\Xi$ defined by (10b), the dependent variable $w(\xi)$ travels on the Riemann surface determined by its dependence on the complex variable $\xi$, as entailed by the equation (11) that relates $w(\xi)$ to its argument $\xi$ - starting at $t=0$ from $\xi=\xi_{0}$,

$$
\xi_{0}=\bar{\xi}+R=(\eta+1) R
$$


(see (9a)- (9d) ) and correspondingly from $w\left(\xi_{0}\right)=w_{0}$,

$$
w_{0}=\frac{1}{\kappa}=\frac{\left[z_{1}(0)-z_{2}(0)\right]^{2}+\left[z_{2}(0)-z_{3}(0)\right]^{2}+\left[z_{3}(0)-z_{1}(0)\right]^{2}}{2 \mu\left[2 z_{3}(0)-z_{1}(0)-z_{2}(0)\right]^{2}} .
$$

We observe that $\xi$ and $\xi_{0}$ feature the same degeneracy as $R$.

We still must fix the degeneracy of the square roots appearing in (6a) $-6 \mathrm{~b}$ ). Since there is no degeneracy in the determination of $w_{0}$ and $\eta$, the determination of the signs of the square roots in (6a)-(6b) is fixed by demanding that these formulae are consistent with the initial data at $t=0$.

Despite the periodicity in time of $\xi$, the corresponding time evolution of $w$ via (11) could be much more complicated (possibly aperiodic) due to the multivaluedness of $w$ as a function of $\xi$. This evolution must be studied by lifting a circular path to the Riemann surface defined by (11). This motivates the necessity to study the geometric structure of the Riemann surface, which we address in the following section.

\section{The Riemann SURface}

In this section we discuss the structure of the Riemann surface $\Gamma$ consisting of points $(\xi, w) \in \Gamma$ such that (11) is satisfied.

For rational values of $\mu=p / q$, which is the case if both the coupling constants $f$ and $g$ are rational numbers, see (5), equation (11) describes an algebraic curve. We treat the case of irrational $\mu$ via an appropriate limit of the case with rational $\mu$. Moreover, as already pointed out in [23] and as we recall in the next section, one needs to distinguish among the three cases with $\mu<0,0<\mu<1$ and $\mu>1$.

We start the analysis of the structure of $\Gamma$ by ignoring the dependence of $\xi$ on the "physical" time $t$ (see (10b)), and by considering instead $\xi$ as an independent complex variable, evolving on a generic (possibly closed) path on the complex $\xi$-plane; only after having thereby obtained an appropriate understanding of the topological properties of $\Gamma$, we proceed and analyze the consequences of the "physical movement" of $\xi$ along the circle $\Xi$, as described by (10b).

3.1. Movable and fixed singularities. The function $w(\xi)$ defined by (11) features two types of singularities: the "fixed" ones occurring at values of the independent variable $\xi$ - and correspondingly of the dependent variable $w$ - that can be read directly from the structure of (11); and the "movable" ones occurring at values of the independent and dependent variables, $\xi$ and $w$, that cannot be directly read from the structure of (11) (they "move" as the initial data are modified).

In order to investigate the nature of the movable singularities, it is convenient to differentiate (11), thereby obtaining (by repeated use of (11))

$$
\xi w^{\prime}=-\frac{w(w-1)}{w-\mu},
$$

where the prime indicates differentiation with respect to $\xi$. The position of the movable singularities, $\xi_{\mathrm{b}}$, and the corresponding values of the dependent variable, $w_{b} \equiv w\left(\xi_{\mathrm{b}}\right)$, are then characterized by the vanishing of the denominator in the right-hand side of this formula, yielding the relation

$$
w_{b}=\mu,
$$

which, combined with (11) (at $\xi=\xi_{\mathrm{b}}$ ) is easily seen to yield

$$
\begin{gathered}
\xi_{\mathrm{b}}=\xi_{\mathrm{b}}^{(k)}=r \exp (2 \pi i \mu k), \quad k=1,2,3, \ldots \\
r=(\mu-1)^{-1}\left(\frac{\mu-1}{\mu}\right)^{\mu} .
\end{gathered}
$$

In (14c) it is understood that the principal determination is to be taken of the $\mu$-th power appearing in the right-hand side. Formula (14b) shows clearly that the number of these branch points is infinite if the parameter $\mu$ is irrational $(\mu \notin \mathbb{Q})$, and that they then sit densely on the circle $B$ in the complex $\xi$-plane centered at the origin and having radius $r$, see (14c). On the contrary, if $\mu$ is rational $(\mu \in \mathbb{Q})$ the branch points sit again on the circle $B$ in the complex $\xi$-plane, but there are only a finite number of them. As proved in 23, these movable singularities are all square-root branch points.

Then, let us consider the "fixed" singularities, which clearly can only occur at $\xi=\infty$ and at $\xi=0$ with corresponding values for $w$. 
Two behaviors of $w(\xi)$ are possible for $\xi \approx \infty$, depending on the value of (the real part of) $\mu$. The first is characterized by the ansatz

$$
w(\xi)=a \xi^{\beta}+o\left(|\xi|^{\beta}\right), \quad \beta<0,
$$

and its insertion in (11) yields

$$
\beta=-\frac{1}{\mu}, \quad a^{\mu}=-\exp (i \pi \mu)
$$

which is consistent with (15a) iff

$$
\mu>0
$$

The second is characterized by the ansatz

$$
w(\xi)=1+a \xi^{\beta}+o\left(|\xi|^{\beta}\right), \quad \beta<0,
$$

and its insertion in (11) yields

$$
\beta=\frac{1}{\mu-1}, \quad a^{\mu-1}=1,
$$

which is consistent with (16a) iff

$$
\mu<1 \text {. }
$$

We therefore conclude that there are only three possibilities:

- if $\mu>1$, only the first ansatz, (15a)-(15c), is applicable, and it characterizes the nature of the branch point of $w(\xi)$ at $\xi=\infty$;

- if $\mu<0$, only the second ansatz, (16a)-(16c), is applicable, and it characterizes the nature of the branch point of $w(\xi)$ at $\xi=\infty$;

- if $0<\mu<1$, both ansätze, (15a)-(15c) and (16a)-(16c), are applicable, so both types of branch points occur at $\xi=\infty$.

The special cases $\mu=0$ and $\mu=1$ require a separate treatment 33. Next, let us investigate the nature of the singularity at $\xi=0$. Two behaviors are possible: either

$$
\begin{gathered}
w(\xi)=a \xi^{\beta}+o\left(|\xi|^{\beta}\right), \quad \beta>0, \\
\beta=-\frac{1}{\mu}, \quad a^{\mu}=-\exp (i \pi \mu),
\end{gathered}
$$

which is applicable if and only if

$$
\mu<0
$$

or

$$
\begin{gathered}
w(\xi)=1+a \xi^{\beta}+o\left(|\xi|^{\beta}\right), \quad \beta>0, \\
\beta=\frac{1}{\mu-1}, \quad a^{\mu-1}=1,
\end{gathered}
$$

which is applicable if and only if

$$
\mu>1 \text {. }
$$

This analysis shows that the function $w(\xi)$ features a branch point at $\xi=0$ the nature of which is characterized by the relevant exponent $\beta$, see (17b) or (18b), whichever is applicable (see (17c) and (18c)). There is no branch point at all at $\xi=0$ if neither one of the two inequalities (17c) and (18c) holds, namely if $0<\mu<1$.

Moreover, we observe that the case $\mu<0$ can be immediately worked out from the case $\mu>1$ via the following replacement

$$
w \mapsto w-1, \quad \xi \mapsto-\xi, \quad \mu \mapsto 1-\mu .
$$

For this reason, only the two cases $0<\mu<1$ and $\mu>1$ lead to different Riemann surfaces. Although the geometrical properties of the Riemann surface are quite different in the two cases, the techniques employed in their study are essentially the same. For this reason, to avoid unnecessary repetitions we concentrate in 
this paper only on the first case $0<\mu<1$, which, as we shall see, leads to a rich and complex behavior. The second case $\mu>1$ which also has interesting consequences for the dynamics, specially in the case $\mu \notin \mathbb{Q}$, shall be discussed in a subsequent paper.

3.2. The case $0<\mu<1$ and $\mu \in \mathbb{Q}$. If $\mu$ is a rational number in the open interval $(0,1)$, namely if

$$
\mu \in \mathbb{Q} \quad \text { and } \quad 0<\mu=\frac{p}{q}<1,
$$

where $p \in \mathbb{N}$ and $q \in \mathbb{N}^{+}$are coprime natural numbers, then (11) implies that $\Gamma$ is an algebraic Riemann surface characterized by the polynomial equation

$$
(w-1)^{q-p} w^{p} \xi^{q}=1 \quad \text { with } \quad 0<p<q,
$$

which defines the $q$-valued function $w=w(\xi)$. Since, $\forall \xi \in \mathbb{C}$, the polynomial (21) admits $q$ complex roots and each root corresponds to a sheet of the Riemann surface $\Gamma$, it follows that $\Gamma$ is a $q$-sheeted covering of the complex $\xi$-plane.

Condition (20), via formulae (15a)-(15c) and (16a)-116c), entails that, for $\xi \approx \infty$, the $\infty$-configuration of the $q$ roots of (21) consists of $p$ roots lying on a small circle of radius $O\left(|\xi|^{-\frac{q}{p}}\right)$ around the origin, and of $(q-p)$ roots lying on a small circle of radius $O\left(|\xi|^{-\frac{q}{q-p}}\right)$ around 1 (see Figure 1). From the point of view of the Riemann surface, we see that, at $\xi=\infty$, the branch point $(\infty, 0)$, of order $(p-1)$, connects $p$ sheets and the branch point $(\infty, 1)$, of order $(q-p-1)$, connects $(q-p)$ sheets.

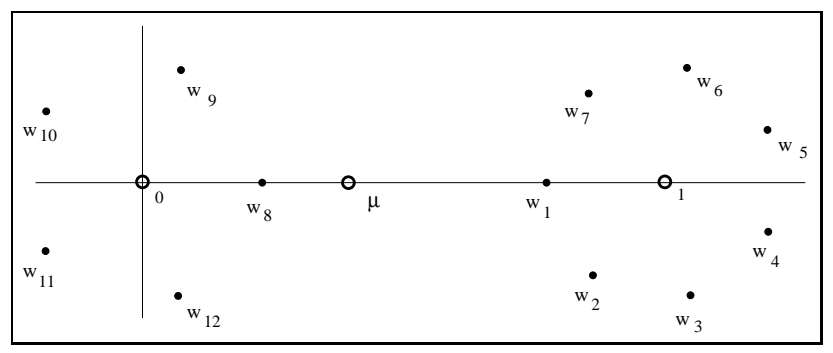

Figure 1. The $\infty$-configuration of the $q$ roots of (21), for $q=12$ and $p=5$. The labeling of the roots is explained in the text.

In the finite part of $\Gamma$, condition (20) and formulae (14a)-(14c) imply that there are only $q$ square-root branch points:

$$
\left(\xi_{\mathrm{b}}^{(j)}, \mu\right) \in \Gamma, \quad j=1, \ldots, q,
$$

defined by the equation:

$$
\xi^{q}=\frac{(-)^{q-p} q^{q}}{p^{p}(q-p)^{q-p}} .
$$

These $q$ square-root branch points correspond to the collision of a pair of roots of (21); they are clearly located on the circle $B$ in the complex $\xi$-plane centered at the origin and having radius $r_{b}$

$$
r_{b}=\frac{q}{q-p}\left(\frac{q-p}{p}\right)^{\frac{p}{q}}>0 .
$$

It is convenient to sort these $q$ square-root branch points sequentially in counterclockwise order (see Figure 2); an opportune choice of the first square-root branch point $\xi_{\mathrm{b}}^{(1)}$, clearly arbitrary at this stage, is suggested by the direct problem and will be discussed later.

The genus of $\Gamma$ is 0 ; this is an immediate consequence of the Hurwitz formula, $V=2(J+G-1)$, where $V$ is the ramification index of the surface, $J$ is the number of sheets and $G$ is its genus. In our case: $J=q$ and $V=q+(q-p-1)+(p-1)=2(q-1)$, entailing $G=0$.

Moreover, equation (21) exhibits several symmetries. The ones used below are: 


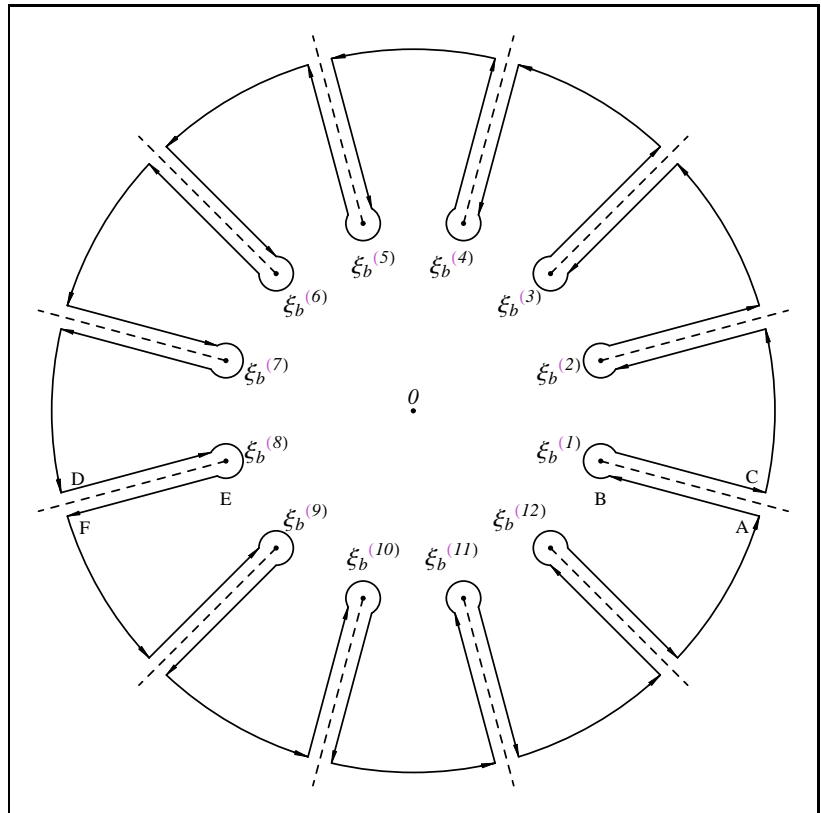

Figure 2. The cut $\xi$-plane of the Riemann surface $\Gamma$ with $q=12$ and $p=5$ is the interior of the oriented contour $\gamma$.

- Symmetry under a $2 \pi / q$ rotation. The set of roots is left invariant by a rotation around the origin of the $\xi$-plane by an angle $2 \pi / q$ (and of course any integer multiple of it).

- Symmetry along the cuts. If $\xi$ belongs to the rays passing through the square-root branch points, (22b) implies that (21) is a polynomial equation featuring real coefficients. Therefore, its roots are real or in complex conjugate pairs. Consider the semiline cuts $\gamma_{j}, j=1, \ldots, q$, defined by

$$
\gamma_{j}=\left\{\xi, \arg \xi=\arg \xi_{\mathrm{b}}^{(j)},|\xi| \geq\left|\xi_{\mathrm{b}}^{(j)}\right|\right\}, \quad j=1, \ldots, q .
$$

Then, if $\xi \in \gamma_{j}, j=1, \ldots, q$, two of the $q$ roots lie on the segment $(0,1)$. If, in addition, $\xi \approx \infty$, then one of these two roots belongs to the small circle around the origin and the other one belongs to the small circle around 1 (see Figure 1, where the $\infty$-configuration of the $q$ roots is shown for $\xi \approx \infty$ and $\left.\xi \in \gamma_{1}\right)$.

3.3. Roots dynamics and topological properties. In order to understand the topological properties of the Riemann surface $\Gamma$, we first cut the $\xi$-complex plane along the rays $\gamma_{j}, j=1, \ldots, q$, see (23), from the square root branch points $\xi_{\mathrm{b}}^{(j)}$ to the branch point at $\infty$, and we introduce the closed contour $\gamma$ whose interior is the cut $\xi$-plane thereby obtained (see Figure 2). Let $\mathcal{F}_{j}$ be the Riemann sheet of $\Gamma$ associated to the root $w_{j}$. Our goal is to construct the $q$ images $\mathcal{I}_{j}, j=1, \ldots, q$, of the cut $\xi$-plane, corresponding to the $q$ roots $w_{j}(\xi)$ of (21) $, j=1, \ldots, q$, and to study their connections.

Notation: Here and hereafter, through Sections 3.2 and 5.2, all the indices in the designation of the squareroot branch points $\xi_{\mathrm{b}}^{(j)}$, the roots $w_{j}$, the images $\mathcal{I}_{j}$ and the sheets $\mathcal{F}_{j}$ are defined MÕD $(q)$ via the congruence defined in (2), namely $\xi_{\mathrm{b}}^{(j)} \equiv \xi_{\mathrm{b}}^{(j \mathrm{MO \tilde {D }}(q))}$ or $\mathcal{F}_{j} \equiv \mathcal{F}_{j \text { MõD }(q)}$.

Our strategy will be to start from the $\infty$-configuration, namely the root configuration entailed by setting $\xi \approx \infty$, and to map out the structure of the Riemann surface as $\xi$ is moved in along rays and it is moved around along circles.

Starting with $\xi \in \gamma_{j}$, it is natural to call $w_{j}$ the root lying on the segment $(0,1)$ and belonging to the small circle around 1 . Then $w_{j+1}, \ldots, w_{j+q-p-1}$ are the other roots of this circle, enumerated sequentially in counterclockwise order. Analogously, we denote by $w_{j+q-p}$ the root lying on the segment $(0,1)$ and belonging to the small circle around 0 ; and by $w_{j+q-p+1}, \ldots, w_{j+q-1}$ the other roots of this circle, enumerated sequentially in counterclockwise order (see Figure 1). 
Using the large $\xi$ asymptotics, (15a)-(15c) and (16a)-(16c), and the symmetries of $\Gamma$ described above, we infer the following basic motions.

- As $\xi$ moves along the cut $\gamma_{j}$, from $\infty$ to $\xi_{\mathrm{b}}^{(j)}$, the two roots $w_{j}$ and $w_{j+q-p}$, lying on the segment $(0,1)$, move along it, from the small circles around 0 and 1 to the collision point $\mu$. In addition, being $\xi_{\mathrm{b}}^{(j)}$ a branch point of square-root type, a $2 \pi$ rotation of $\xi$ around it corresponds to a $\pi$ rotation of these two roots around $\mu$. All this implies that, if $\xi$ travels along the contour surrounding the cut $\gamma_{j}$ (for instance, moving from the point $A$ to the point $C$ along the path shown in Figure 2), then the two roots $w_{j}$ and $w_{j+q-p}$ involved in the collision exchange their position (see Figure 3). The remaining roots are essentially unaffected by this motion, moving back and forth on lines and going back to their starting positions. We have thereby established the first basic motion: a motion of $\xi$ around the branch cut $\gamma_{j}$ yields an exchange of the two roots $w_{j}$ and $w_{j+q-p}$.

- If, starting from the cut $\gamma_{j}, \xi$ performs a $2 \pi / q$ counterclockwise rotation, moving from the cut $\gamma_{j}$ to the cut $\gamma_{j+1}$, then the $(q-p)$ roots surrounding 1 undergo a clockwise rotation around 1 , while the $p$ roots surrounding 0 undergo a clockwise rotation around 0 . When $\xi$, starting from $\gamma_{j}$, reaches $\gamma_{j+1}$, two new roots belonging to the two small circles get aligned on the segment $(0,1)$; they are just $w_{j+1}$ and $w_{j+1+q-p}$ (see Figure 4). Therefore the two sets of roots undergo cyclic permutations, which is the second basic motion: a rotation of $\xi$ from $\gamma_{j}$ to $\gamma_{j+1}$ produces a cyclic permutation of the two sets of roots in the $\infty$-configuration $\left\{w_{1}, \ldots, w_{q-p}\right\}$ and $\left\{w_{q-p+1}, \ldots, w_{q}\right\}$.

Repeating $q$ times the above two motions with respect to the other cuts, in sequential order, the point $\xi$ draws the whole closed contour $\gamma$ and correspondingly, due to the above-mentioned basic motions and symmetries, each root $w_{j}$ draws the closed contour in Figure 5 around the cut $[0,1]$ of the $w$-plane. The following details can be given (compare Figure 2 and Figure 5 ).

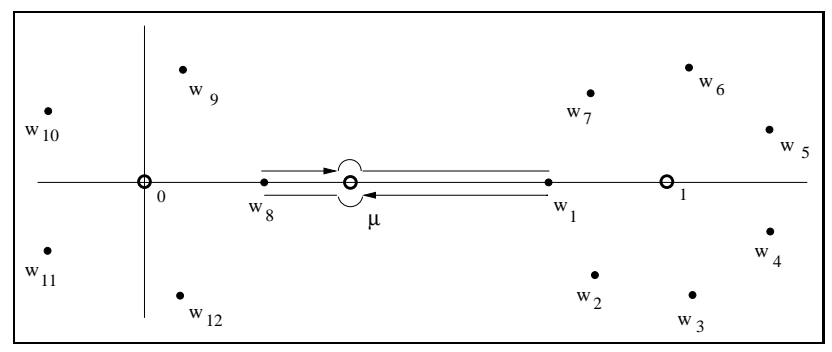

FiguRE 3. Exchange of a pair of roots. As $\xi$ travels on the contour surrounding the cut $\gamma_{j}$ (for example moving from the point $A$ to the point $C$ along the path shown in Figure 21), $w_{j}$ and $w_{j+q-p}$ interchange their positions, while the other $(q-p)$ roots are essentially unaffected by this motion.

(1) As $\xi$ moves on $\gamma$ through the points $A, B$ and $C$, around the cut $\gamma_{j}, w_{j}$ moves along the cut $[0,1]$ through the homologous points, exchanging its position with the root $w_{j+q-p}$.

(2) As $\xi$ moves on $\gamma$ from $C$ to $D$, the relevant part of the motion of $w_{j}$ consists in a clockwise rotation around 0 , from $C$ to $D$.

(3) As $\xi$ moves on $\gamma$ through the points $D, E$ and $F$, around the cut $\gamma_{j-q+p}, w_{j}$ moves along the cut $[0,1]$ through the homologous points, exchanging its position with the root $w_{j-q+p}$.

(4) As $\xi$ completes the contour $\gamma$, moving from $F$ to the starting point $A$, also $w_{j}$ completes its closed contour around $[0,1]$, and the relevant part of this motion consists in a clockwise rotation around 0 , from $F$ to $A$.

From the above considerations we finally infer the following

Topological properties of $\Gamma$ for $\mu=\mathbf{p} / \mathbf{q}$ and $\mathbf{0}<\mathbf{p}<\mathbf{q}$. The Riemann surface $\Gamma$ defined in (21) is a $q$-sheeted covering of the $\xi$-plane of genus 0 . In the finite part of $\Gamma$ there are $q$ square-root branch points: $\left(\xi_{\mathrm{b}}^{(j)}, \mu\right) \in \Gamma, j=1, \ldots, q$ (see (23)). If $\mathcal{F}_{j}$ is the sheet associated to the root $w_{j}$, then the $j$-th branch point connects the sheets $\mathcal{F}_{j}$ and $\mathcal{F}_{j+q-p}$. Each sheet $\mathcal{F}_{j}$ contains just the two square-root branch points $\left(\xi_{\mathrm{b}}^{(j)}, \mu\right)$ and $\left(\xi_{\mathrm{b}}^{(j-q+p)}, \mu\right)$. The compactification of $\Gamma$ is achieved at $\xi=\infty$, where the branch point $(\infty, 1)$, of order 


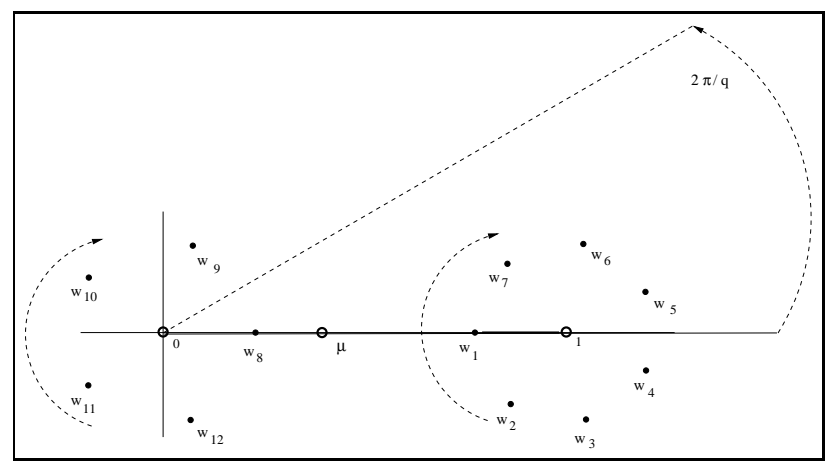

FIgURE 4. Cyclic permutation of the two groups of roots. If $\xi \in \gamma_{j}$, the roots $w_{j}$ and $w_{j+q-p}$ are aligned on the segment $(0,1)$; after a $2 \pi / q$ counterclockwise rotation of $\xi$, from the cut $\gamma_{j}$ to the cut $\gamma_{j+1}$, the two groups of roots $\left\{w_{j}, . ., w_{j+q-p-1}\right\}$ and $\left\{w_{j+q-p-1}, . ., w_{j+q-1}\right\}$ undergo a clockwise rotation. When $\xi$ reaches $\gamma_{j+1}$, then the roots $w_{j+1}$ and $w_{j+1+q-p}$ get aligned on the segment $(0,1)$.

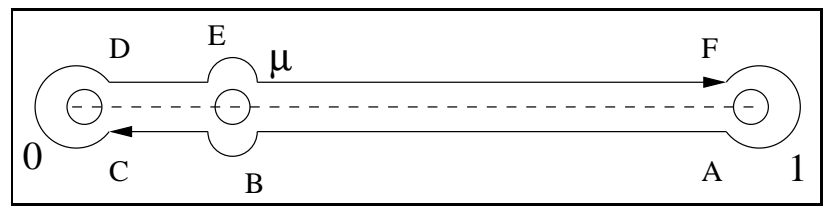

FiguRE 5. As $\xi$ travels on the closed contour $\gamma$ in Figure 2 each root travels on a closed contour around the cut $[0,1]$ in the $w$-plane. Therefore the image of the cut $\xi$-plane is the $w$-plane cut along the segment $[0,1])$.

$(q-p-1)$, connects the first $(q-p)$ sheets, and where the branch point $(\infty, 0)$, of order $p-1$, connects the remaining $p$ sheets. As $\xi$ turns counterclockwise around $(\infty, 1)$, the connected sheets are visited in the order: $\mathcal{F}_{j}, \mathcal{F}_{j+p}, \mathcal{F}_{j+2 p}, \ldots ;$ as $\xi$ turns counterclockwise around $(\infty, 0)$ instead, the connected sheets are visited in the order: $\mathcal{F}_{j}, \mathcal{F}_{j+q-p}, \mathcal{F}_{j+2(q-p)}, \ldots$

3.4. The physical root. Once a labeling system has been introduced for the square-root branch points $\xi_{\mathrm{b}}^{(j)}$ 's and for the roots $w_{j}$ 's, in order to solve our original problem (11) we need to indicate which of the $q$ roots of (21) corresponds to the physical root $\check{w}(t) \equiv w(\xi)$ appearing in (6a)-(6b) and satisfying (8).

As implied by (12a) and (12b), at $t=0$ we have that $\xi=\xi_{0}$ and one of the $q$ roots of (21) is $w_{0}$. Therefore, it is sufficient to label $w_{0}$ in order to label the physical root. Observing (12a) and (9b), we immediately note that $\xi_{0}$ is a $q$-valued function of the initial conditions $z_{1}(0), z_{2}(0)$ and $z_{3}(0)$; on the contrary, from (12b) we infer that $w_{0}$ is a single-valued function of the initial conditions $z_{1}(0), z_{2}(0)$ and $z_{3}(0)$. Via (9c) and (10b), we get that $\xi_{0}$ and $\bar{\xi}$, the center of the circle $\Xi$ on which the $\xi$ variable moves, have the same degeneracy. This degeneracy can be removed by arbitrarily fixing one of the $q$ determinations of $R$ in (9b), placing the circle $\Xi$ on the $\xi$-plane and then labeling counterclockwise the square-root branch points lying on the circle $B$ defined by (22b) so that $\xi_{\mathrm{b}}^{(1)}$ is the first branch point encountered by moving counterclockwise on the arc of the circle $B$ obtained by intersecting the two circles and contained inside the circle $\Xi$ (see Figure 6 ). When the two circles $\Xi$ and $B$ do not intersect, then the first branch point $\xi_{\mathrm{b}}^{(1)}$ on $B$ can be chosen arbitrarily.

To proceed in labeling the physical root, we rephrase (21) in the following form

$$
\left[(x-1)^{2}+y^{2}\right]^{\frac{q-p}{2}}\left(x^{2}+y^{2}\right)^{\frac{p}{2}}|\xi|^{q} e^{i[q \arg (\xi)+(q-p) \arg (x-1+i y)+p \arg (x+i y)]}=1,
$$

where $x=\Re(w)$ and $y=\Im(w)$ are respectively the real and imaginary part of $w$ and $\arg (\xi)$ is the argument of the complex variable $\xi$. Equation (24) implies that, for fixed $\xi$ and for rational values of $\mu$, all the roots of (21) lie on the algebraic curve on the $w$-plane given in implicit form by

$$
\left[(x-1)^{2}+y^{2}\right]^{q-p}\left(x^{2}+y^{2}\right)^{p}=|\xi|^{-2 q} .
$$




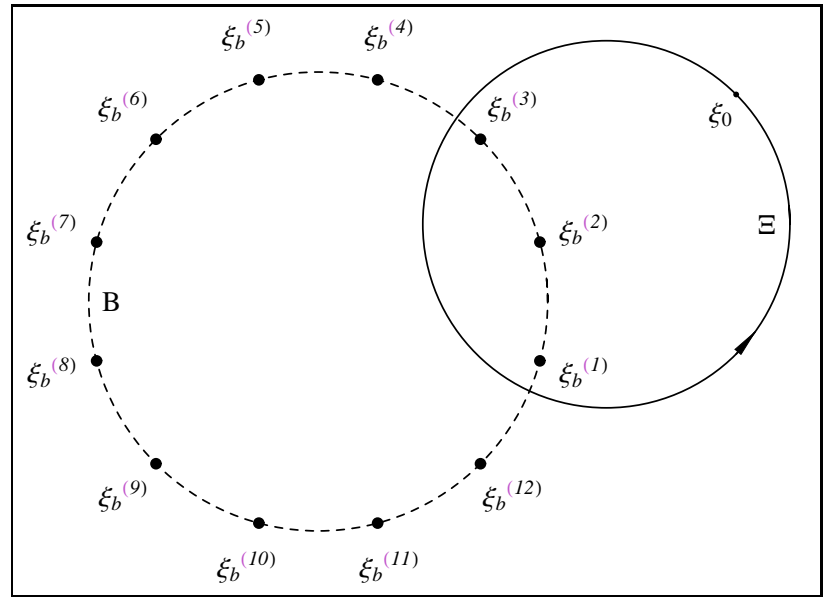

FiguRE 6 . The variable $\xi$ moves counterclockwise on the circle $\Xi$ starting from $\xi_{0}$. The first square-root branch point on the circle $B$ is the first one encountered by moving counterclockwise on the arc of $B$, obtained by intersecting the two circles and contained inside the circle $\Xi$. In this example $p=5$ and $q=12$.

As implied by the discussion in Section 3.2, for $|\xi|<r_{b}$ the curve (25) consists of a single closed branch that becomes a big circle of radius $o\left(|\xi|^{-1}\right)$ around the origin of the $w$-plane as $|\xi| \rightarrow 0$; for $|\xi| \geq r_{b}$ the curve (25)) consists of two closed branches that become two small circles, one of radius $O\left(|\xi|^{-\frac{q}{p}}\right)$ around the origin and the other of radius $O\left(|\xi|^{-\frac{q}{q-p}}\right.$ ) around 1 , as $|\xi| \rightarrow \infty$ (see Figure 7).

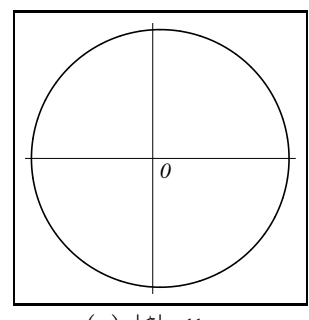

(a) $|\xi| \ll r_{b}$

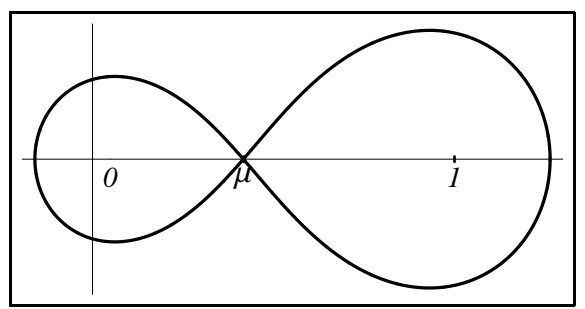

(c) $|\xi|=r_{b}$

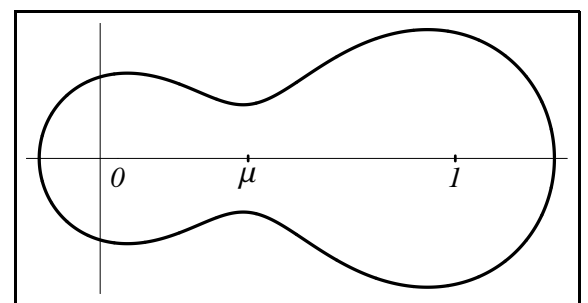

(b) $|\xi|<r_{b}$

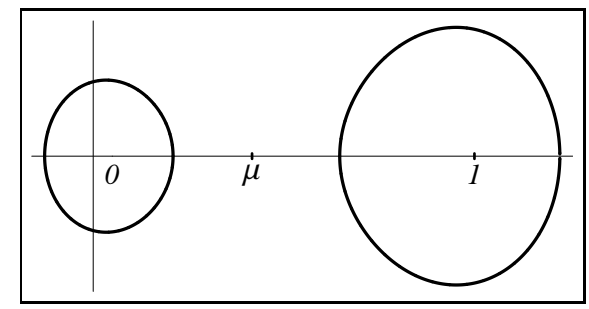

(d) $|\xi|>r_{b}$

Figure 7. The locus of the roots (25) on the $w$-plane for different values of $|\xi|$ when $p=5$ and $q=12$.

If the independent variable $\xi$ moves counterclockwise on a circle around the origin, namely if only the phase of $\xi$ varies, then the roots of (21) move clockwise on the curve (25), undergoing cyclic permutations: if (25) is constituted by two unconnected branches, then the two systems of roots undergo two separate cyclic permutations.

On the other hand, (22b) implies that, if $\xi$ moves along one of the rays that start at the origin of the $\xi$-plane and intersect the branch points, i.e. if $\arg (\xi)=\arg \left(\xi_{\mathrm{b}}^{(j)}\right)$ for some $j$, then $\xi^{q} \in \mathbb{R}$. Therefore, via (24), one immediately gets that in this case all the roots of (21) lie on the $w$-plane on the locus defined in 
implicit form by

$$
\begin{gathered}
(q-p) \arg (x-1+i y)+p \arg (x+i y)=n \pi, \\
\text { with }\left\{\begin{array}{ll}
n=1,3,5, \ldots, 2 q-1 \\
n=0,2,4, \ldots, 2 q-2
\end{array} \text { if }(q-p) \text { is odd }(q-p) \text { is even } .\right.
\end{gathered}
$$

The branches of the curve (26) define a partition of the $w$-plane (see Figure 8). In other words, the partition of the $\xi$-plane into the $q$ sectors obtained by tracing the rays that start at the origin and cross the square-root branch points (see Figure 9(a) induces a partition of the $w$-plane into the $q$ sectors individuated by (26): if the variable $\xi$ moves entirely in a single angular sector on the $\xi$-plane, without crossing the lines that define the sectors, then each root of (21) is contained in a single region of the $w$-plane, without crossing any of the branches of the curve (26) that separate the regions.

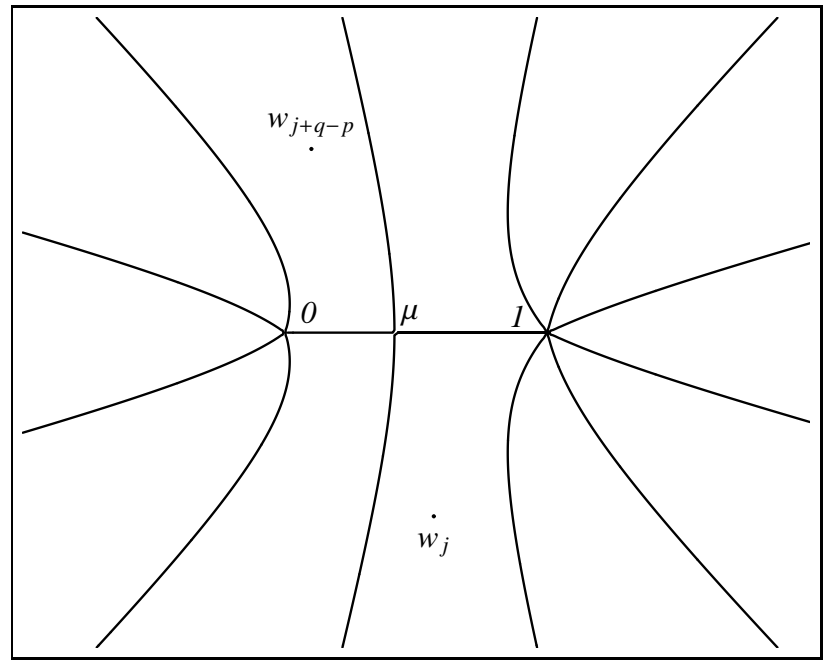

Figure 8. The curve (26) divides the $w$-plane in $q$ sectors. For a fixed value of $\xi$, each root of (21) lies inside a sector. When $\xi$ moves counterclockwise around the cut $\gamma_{j}$, the roots $w_{j}$ and $w_{q-p+j}$ (that are respectively in the lower and upper exchange region) exchange their positions in the corresponding sectors. In this example $p=5$ and $q=12$.

For $q \geq 3$ there is always a branch of the curve (26) that intersects the point $\mu$ on the $w$-plane. As shown in the previous section, when $\xi$ moves around the branch cut $\gamma_{j}$ then the two roots $w_{j}$ and $w_{j+q-p}$ exchange their positions moving around the point $\mu$ on the $w$-plane (see Figure 8). Therefore, if the root $w_{j}$ is contained in the lower half-plane of the $w$-plane in the region that is bounded by the real segment $(\mu, 1)$ and by the lower branch of the curve (26) that crosses the point $\mu$ (the lower exchange region), then the root $w_{j+q-p}$ is contained in the upper half-plane of the $w$-plane in the region that is bounded by the real segment $(0, \mu)$ and by the upper branch of the curve (26) that crosses the point $\mu$ (the upper exchange region).

We assign to each angular sector on the $\xi$-plane the same label of the branch point that is next to the sector (counterclockwise), see Figure 9(a) If the initial datum $\xi_{0}$ is contained in the sector $j$ on the $\xi$-plane, then correspondingly the root $w_{j}$ is in the lower exchange region on the $w$-plane. Once a label has been assigned to the lower exchange region, we can label all the remaining regions (and correspondingly the roots in them) by counting them counterclockwise MõD $(q)$, see Figure 9(b). When all the regions on the $w$-plane have been labeled, then the label of the physical root is the label of the region where $w_{0}$ falls.

The prescription to label the physical root can be summarized as follows (see Figure 9):

(1) Trace the circle $B$ in the $\xi$-plane, on which the branch points lie (see (14)).

(2) Choose arbitrarily the same determination for $\xi_{0}$ and $R$ (see (12a)).

(3) Trace the circle $\Xi$ (see (10b) ) and select, as explained in the present section, the first branch point $\xi_{\mathrm{b}}^{(1)}$. Starting from there, label the remaining branch points counterclockwise. Define the angular sectors on the $\xi$-plane.

(4) Compute $\xi_{0}$ and put it on the $\xi$-plane. Denote by $\hat{j}$ the sector where $\xi_{0}$ falls. 


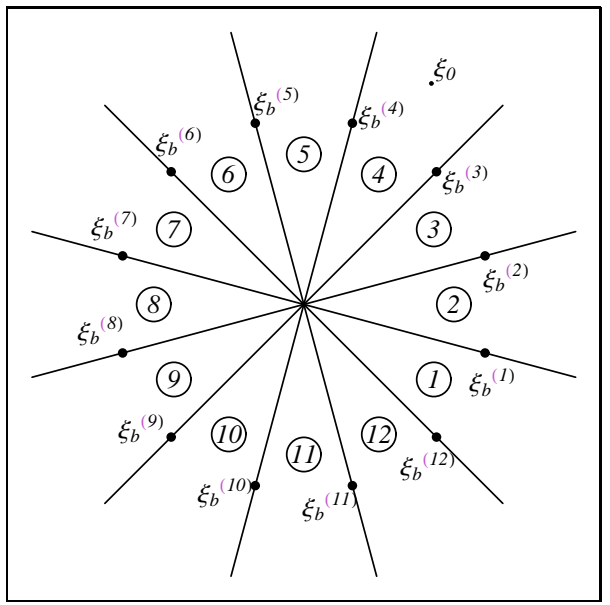

(a) Sector labeling of the $\xi$-plane.

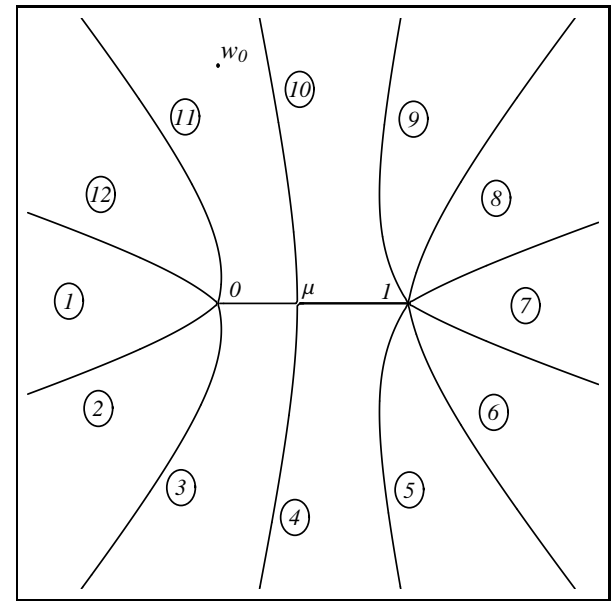

(b) Corresponding sector labeling of the $w$ plane.

Figure 9. The enumeration scheme for the roots of (21). In this example $p=5$ and $q=12$, $\xi_{0}$ is in the fourth sector on the $\xi$-plane, while the physical root $w_{0}$ lies in the eleventh sector on the $w$-plane.

(5) Trace the curve (26) on the $w$-plane. Enumerate the regions defined by the branches of the curve by assigning MÕD $(q)$ the label $\hat{j}$ to the lower exchange region and moving counterclockwise.

(6) From the initial data, compute $w_{0}$ and put it on the $w$-plane. The label of the physical root is the label of the region where $w_{0}$ falls.

This mechanism to identify the physical zero cannot be applied when $\mu$ is irrational as the separatrices of the sectors become infinitely close. Therefore this case requires a separate treatment.

\section{Connection With GRAPH THEORY}

In this section we apply graph theory in order to describe the monodromy group associated to (21), namely the subgroup of the symmetrical group $\mathcal{S}_{q}$ corresponding to all possible exchanges and cyclic permutations occurring on the system of $q$ roots when the independent variable $\xi$ moves along a closed path on the $\xi$-plane. Since eventually $\xi$ shall travel along the circle $\Xi$ described by $(10 \mathrm{~b})$ and the square-root branch points lie on the circle $B$, see (22C), we only need to consider the inclusions of (one or more) consecutive square-root branch points on $B$.

We associate a labeled planar graph $V$ to each mutual configuration of the two circles $\Xi$ and $B$. In order to construct this graph, let us trace $q$ nodes - corresponding to the sheets of the Riemann surface $\Gamma$ - on the vertices of a regular polygon; let us arbitrarily give to one of the nodes the label 1 ; if $v_{j}$ is the label of the $j$-th node of the graph, counting counterclockwise from the node 1 , then the labels are given by the following rule: $v_{1}=1$ and $v_{j+1}=\left(v_{j}+q-p\right)$ MÕD $(q), j=1, \ldots, q-1$. When $\Xi$ includes no square-root branch points, then the planar graph has no edges (see Figure 10(a)p. If the circle $\Xi$ includes the square-root branch point $\xi_{\mathrm{b}}^{(j)}, j=1, \ldots, q$, correspondingly the planar graph gets an edge between the nodes $j$ and $(j+q-p)$ MÕD $(q)$, as illustrated in Figure 10. With this prescription, $V$ depends on $\Xi, p$ and $q$, namely $V \equiv V(\Xi, p, q)$.

From the general theory we know that the inclusion of exactly $q$ square-root branch points inside the circle $\Xi$ is equivalent to the inclusion of the branch point at infinity: in this case the period of the zeros of (21) are $q-p$ or $p$, see (15a)-(15c) respectively (16a)-(16c), with the first $q-p$ roots having period $q-p$ and the last $p$ roots having period $p$. From now on, we will suppose to have at most $q-1$ consecutive square-root branch points, starting from $\xi_{\mathrm{b}}^{(1)}$, inside the circle $\Xi$.

A path of length $n$ on the graph $V$ is a sequence of $n$ adjacent distinct nodes $\left\{v_{j_{1}}, v_{j_{2}}, \ldots, v_{j_{n}}\right\}$, namely such that $\left(v_{j_{1}}, v_{j_{2}}\right),\left(v_{j_{2}}, v_{j_{3}}\right), \ldots,\left(v_{j_{n-1}}, v_{j_{n}}\right)$ are edges of the graph. For fixed $\Xi, p$ and $q$, if in $V(\Xi, p, q)$ the node $v_{j}$ belongs to a path of length $b$, then the period of the corresponding root of the algebraic equation (21), $w_{v_{j}}(\xi)$, is exactly $b$, while $\xi$ moves along the circle $\Xi$. If the node $v_{j}$ is unconnected (i.e. it is touched 


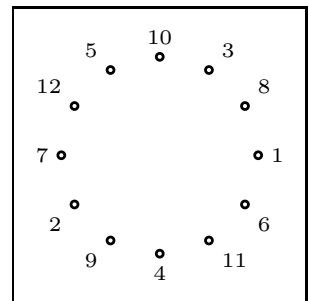

(a) No branch points

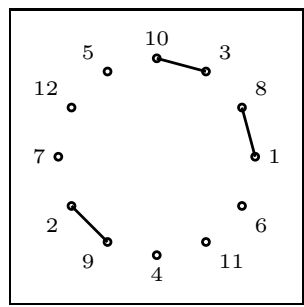

$(\mathrm{d}) \xi_{\mathrm{b}}^{(1)}, \xi_{\mathrm{b}}^{(2)}, \xi_{\mathrm{b}}^{(3)}$

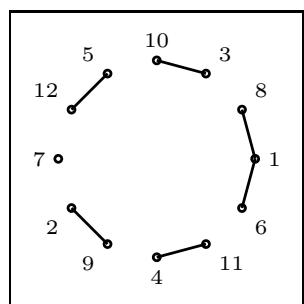

$(\mathrm{g}) \xi_{\mathrm{b}}^{(1)}, \xi_{\mathrm{b}}^{(2)}, \ldots, \xi_{\mathrm{b}}^{(6)}$

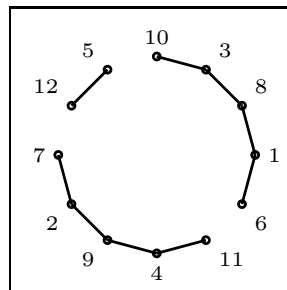

(j) $\xi_{\mathrm{b}}^{(1)}, \xi_{\mathrm{b}}^{(2)}, \ldots, \xi_{\mathrm{b}}^{(9)}$

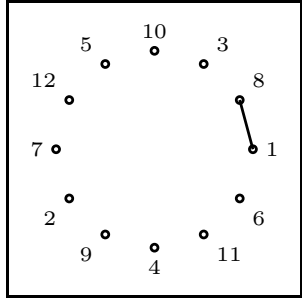

(b) $\xi_{\mathrm{b}}^{(1)}$

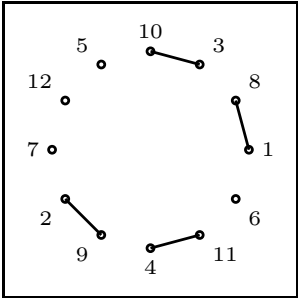

$(\mathrm{e}) \xi_{\mathrm{b}}^{(1)}, \xi_{\mathrm{b}}^{(2)}, \ldots, \xi_{\mathrm{b}}^{(4)}$

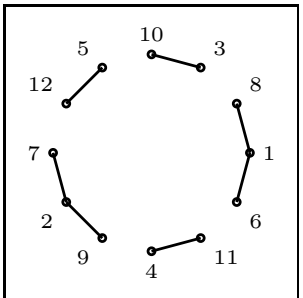

(h) $\xi_{\mathrm{b}}^{(1)}, \xi_{\mathrm{b}}^{(2)}, \ldots, \xi_{\mathrm{b}}^{(7)}$

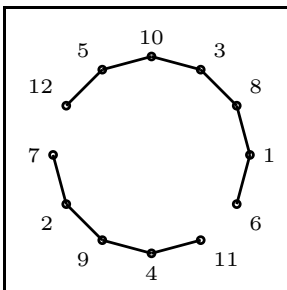

$(\mathrm{k}) \xi_{\mathrm{b}}^{(1)}, \xi_{\mathrm{b}}^{(2)}, \ldots, \xi_{\mathrm{b}}^{(10)}$

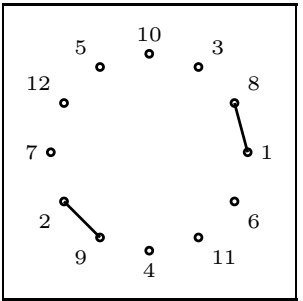

(c) $\xi_{\mathrm{b}}^{(1)}, \xi_{\mathrm{b}}^{(2)}$

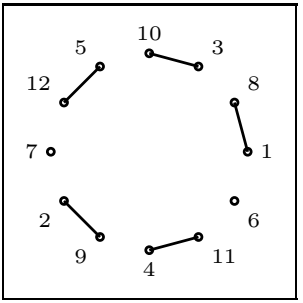

(f) $\xi_{\mathrm{b}}^{(1)}, \xi_{\mathrm{b}}^{(2)}, \ldots, \xi_{\mathrm{b}}^{(5)}$

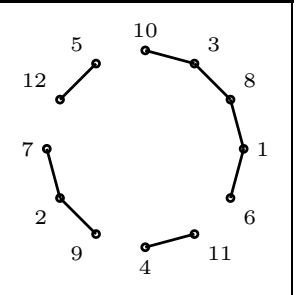

(i) $\xi_{\mathrm{b}}^{(1)}, \xi_{\mathrm{b}}^{(2)}, \ldots, \xi_{\mathrm{b}}^{(8)}$

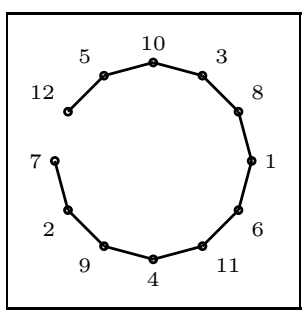

(1) $\xi_{\mathrm{b}}^{(1)}, \xi_{\mathrm{b}}^{(2)}, \ldots, \xi_{\mathrm{b}}^{(11)}$

Figure 10. Planar graphs associated to different inclusions of consecutive square-root branch points in the circle $\Xi$, for $p=5$ and $q=12$. In each caption, the included branch points are indicated.

by no edges), then the period of $w_{v_{j}}(\xi)$ is 1 . For fixed $p$ and $q$ and for a certain mutual configuration of the two circles $\Xi$ and $B$, in order to describe the behavior of the roots of (21), we need to measure the lengths of the paths in the corresponding graph $V(\Xi, p, q)$.

For a graph $V(\Xi, p, q)$ with $q$ nodes, the set of all path lengths corresponds to a decomposition in cycles of elements of the symmetrical group $\mathcal{S}_{q}$. Once the correspondence between the graphs $V$ 's and the elements of $\mathcal{S}_{q}$ has been established, we can use the well-known correspondence between Ferrer diagrams and permutations. We recall that a Ferrer diagram is an ordered disposition of $q$ blank boxes in rows and columns, such that the number of boxes in each column equals the lengths of the cycles of the corresponding permutation and the total number of columns is the number of cycles in which the permutation is decomposed. For instance, the Ferrer diagram 


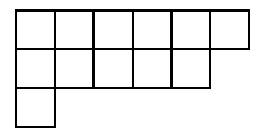

corresponds to the planar graph presented in Figure $10(\mathrm{~g})$. In a Ferrer diagram, the columns are sorted in decreasing order of length, from left to right. Moreover, note that each column in a Ferrer diagram is oriented up-down, the first row being at the top, the last row being at the bottom. Then the problem of knowing the period of a root of equation (21), while $\xi$ moves along the circle $\Xi$, is reduced to measuring the lengths of the columns of an appropriate Ferrer diagram.

4.1. The bumping rule. In this subsection we show how to recursively build the Ferrer diagrams associated to the planar graphs $V$ 's. For fixed $p$ and $q$, suppose that the circle $\Xi$ includes $b$ square-root branch points, so that $V(\Xi, p, q)$ is the corresponding labeled planar graph. In the following, we include only consecutive squareroot branch points of $B$ in $\Xi$, always starting from $\xi_{\mathrm{b}}^{(1)}$ and moving counterclockwise: $\left\{\xi_{\mathrm{b}}^{(1)}, \xi_{\mathrm{b}}^{(2)}, \ldots, \xi_{\mathrm{b}}^{(b)}\right\}$. Under this hypothesis, $V(\Xi, p, q)$ depends on $p$ and $q$ and on $\Xi$ only via the number $b$ of included squareroot branch points. Assuming that $p$ and $q$ have been fixed once for all, we set $V(\Xi, p, q) \equiv V(b)$. Let $F(b)$ indicate the Ferrer diagram corresponding to the planar graph $V(b)$. In what follows, we construct a recursive bumping rule for Ferrer diagrams, namely a rule that permits to build $F(b+1)$ from $F(b)$.

First of all, we need two integer sequences:

$$
\begin{aligned}
q_{k}=p_{k-1}, & q_{0}=q, \\
p_{k}=q_{k-1} \operatorname{MOD}\left(p_{k-1}\right), & p_{0}=q-p,
\end{aligned}
$$

with $0 \leq k \leq \bar{k}$, where $\bar{k}$ is an integer number such that

$$
q_{\bar{k}}=1 ;
$$

note that $\bar{k}$ always exists due to the decreasing nature of the sequence $\left\{q_{k}\right\}$. We need also an auxiliary recursive sequence, written as a combination of the previous two,

$$
b_{k}=b_{k-1}+q_{k-1}-p_{k-1}, \quad b_{0}=0 .
$$

We remark here that the $b$-sequence (28a) divides the discrete segment $[1, q-1]$ into $\bar{k}$ parts of length $q_{k}-p_{k}$. Moreover, from the first of the (27a), via (28a), one gets

$$
\begin{aligned}
b_{0}=0, b_{k} & =b_{k-1}+q_{k-1}-q_{k}=b_{k-2}+q_{k-2}-q_{k-1}+q_{k-1}-q_{k} \\
& =b_{k-2}+q_{k-2}-q_{k}=\ldots=b_{k-h}+q_{k-h}-q_{k} \\
& =b_{0}+q_{0}-q_{k}=q-q_{k} .
\end{aligned}
$$

Suppose that $\Xi$ includes $b$ square-root branch points. If we modify $\Xi$ so that it includes also the next adjacent branch point, correspondingly we modify $V(b)$ into $V(b+1)$, adding to $V(b)$ a new edge between the nodes $(b+1)$ and $(b+1+q-p)$ MÕD $(q)$.

If $0 \leq b<b_{1}-1=q-p-1$, then each new edge added to the graph $V(b)$ connects a single unconnected node to a path composed by a certain number of edges (at least, to another single unconnected node). When $b=b_{1}-1$, it is impossible to trace a new edge on $V(b)$ connecting two unconnected nodes or a single node to a path: the insertion of a new edge in a graph at this point causes the connection of two paths, resulting in a new longer path in the graph $V(b+1)$.

To understand what happens when $b_{1} \leq b<b_{2}-1$, let us imagine to build an auxiliary graph in the following way: associate a weighted planar graph $\tilde{V}(b)$ to the graph $V(b)$, so that, for each path on $V(b)$, you have a weighted node in $\tilde{V}(b)$, with weights on $\tilde{V}(b)$ equal to the lengths of the paths on $V(b)$ (we recall that a single node is equivalent to a path of length 1 ). Now the discussion of the case $b_{1} \leq b<b_{2}-1$ is analogous to the discussion of the previous case $0 \leq b<b_{1}-1$, using $\tilde{V}(b)$ instead of $V(b)$, with the only difference that the length of a path in $\tilde{V}(b)$ is not just the number of nodes touched by the path, but the sum of all the weights of the nodes touched by the path. If $b_{1} \leq b<b_{2}-1$, then each new edge added to the auxiliary graph $\tilde{V}(b)$ connects a single unconnected weighted node to a weighted path composed by a certain number of edges (at least, to another single unconnected weighted node). But when $b=b_{2}-1$, again it is impossible to trace a new edge connecting two unconnected weighted nodes or a single weighted node to a 
weighted path on $\tilde{V}(b)$ : the insertion of a new edge in $\tilde{V}(b)$ at this point will cause the connection of two weighted paths, producing a new longer path in the auxiliary graph.

In order to progressively include more branch points inside $\Xi$, we must build each time a new auxiliary graph, in the afore-described way. We iterate this operation, building a new auxiliary weighted graph every time we arrive to include exactly $b_{k}$ branch points inside the circle $\Xi$, until we reach $b=q-1$. In this language, $q_{k}$ is the number of unconnected weighted nodes in each auxiliary graph when $b_{k} \leq b<b_{k+1}$, while $p_{k}$ is the number of weighted nodes in each auxiliary graph when exactly $b_{k+1}$ branch points are included in $\Xi$.

The use of Ferrer diagrams strongly simplifies this picture. When $b=b_{k}$, the lengths of the columns of the Ferrer diagram $F(b)$ represent the values of the weights of the nodes of the corresponding auxiliary weighted graph. If $b_{k}<b<b_{k+1}$, then the lengths of the columns of the corresponding Ferrer diagrams $F(b)$ represent the lengths of the weighted paths in the corresponding auxiliary weighted graphs. When $b=b_{k}$, the number of columns in $F(b)$ is $q_{k}$. Moreover, note that, if one starts to count columns from the left in $F(b)$, when $b_{h} \leq b<b_{h+1}$, then the columns lying at the positions $p_{h}+1, p_{h}+2, \ldots$, have all the same length, since they coincide with the shortest paths on the corresponding planar graphs.

If the circle $\Xi$ includes $b$ consecutive square-root branch points, when $b_{j} \leq b<b_{j+1}$, and if we modify it in order to include $b+1$ consecutive square-root branch points, then we can build $F(b+1)$ just moving the whole last column on the right of the Ferrer diagram $F(b)$ under the first available column from the left, never occupying positions beyond the $p_{j}$-th column. To pass from $b_{j}$ to $b_{j+1}$, see (28a), we have to move $q_{j}-p_{j}$ columns. All the above considerations lead to the following prescription.

The bumping rule. The Ferrer diagram $F(0)$, corresponding to the inclusion of zero branch points, is composed by a single row of $q$ columns of length 1 . The Ferrer diagram $F(b)$, with $b \leq q-1$, can be obtained from the Ferrer diagram $F(b-1)$ in the following way:

- compute $h$ such that $b_{h} \leq b<b_{h+1}$;

- counting the columns of $F(b-1)$ from the left, move its whole column located at the position $p_{h}+1$ under the column located at the position $\left(b-b_{h}\right)$ MÕD $\left(p_{h}\right)$.

We remark that, in principle, one could ask to move any column lying at the position $p_{h}+1, p_{h}+2, \ldots$, since they all have the same length. The reason for prescribing a movement of the column lying at the position $p_{h}+1$ will be clear after the introduction in Subsection 5.1 of a numeration for the blank boxes of the Ferrer diagrams.

For each $k$ between 0 and $\bar{k}$, (27c), the Ferrer diagram $F\left(b_{k}\right)$ features $q_{k}$ columns of two possible lengths only, $p_{k}$ columns of length $T_{k}^{(1)}$ and $q_{k}-p_{k}$ columns of length $T_{k}^{(2)}$ (see the diagrams in Figure 11, neglecting the numeration, that will be explained below). A $k$-level Ferrer diagram is a Ferrer diagram with exactly $q_{k}$ columns.

The bumping rule implies that we "pass" from a $k$-level Ferrer diagram to a $(k+1)$-level Ferrer diagram once we have moved all the shortest columns on the right under the tallest columns on the left.

The column lengths, $T_{k}^{(1)}$ and $T_{k}^{(2)}$, in a $k$-level Ferrer diagram can be given by the following recursive rule:

$$
\begin{cases}T_{k}^{(1)} p_{k}+T_{k}^{(2)}\left(q_{k}-p_{k}\right)=q_{0}, & T_{0}^{(1)}=1, \\ T_{k+1}^{(1)}=T_{k}^{(1)}+T_{k}^{(2)}\left(\left\lfloor\frac{q_{k}}{p_{k}}\right\rfloor+1\right), & T_{0}^{(2)}=1,\end{cases}
$$

where $\lfloor x\rfloor$ is the floor of the number $x$ (namely, the largest integer less than or equal to $x$ ). Indeed, the first of the (29) is a sort of conservation rule for the number of boxes in a $k$-level Ferrer diagram. The second of the (29) comes directly from the bumping rule: at each level, the length of the first column is the sum of the length of the column at the previous level plus the length of the last column multiplied by the number of the necessary column movements. Although relations (29) are enough to determine the two quantities $T^{(1)}$ and $T^{(2)}$, it is more convenient to put them in a different form:

$$
\begin{cases}T_{k+1}^{(1)}=T_{k}^{(1)}+T_{k}^{(2)}\left(\left\lfloor\frac{q_{k}}{p_{k}}\right\rfloor+1\right), & T_{0}^{(1)}=1, \\ T_{k+1}^{(2)}=T_{k}^{(1)}+T_{k}^{(2)}\left(\left\lfloor\frac{q_{k}}{p_{k}}\right\rfloor\right), & T_{0}^{(2)}=1 .\end{cases}
$$




\begin{tabular}{|c|c|c|}
\hline \begin{tabular}{|l|l|l|l|l|l|l|l|l|l|l|}
1 & 2 & 3 & 4 & 5 & 6 & 7 & 8 & 9 & 10 & 11 \\
\end{tabular} & \begin{tabular}{|l|l|l|l|l|l|l|l|l|l|}
1 & 2 & 3 & 4 & 5 & 6 & 7 & 9 & 10 & $11 \mid 12$ \\
8 & & & & & & & \\
\end{tabular} & \begin{tabular}{|l|l|l|l|l|l|l|l|}
1 & 2 & 3 & 4 & 5 & 6 & 7 & $10|11| 1$ \\
8 & 9 & & & & \\
\end{tabular} \\
\hline
\end{tabular}
(a) $b=b_{0}=0$
(b) $b=1$
(c) $b=2$

\begin{tabular}{|c|c|}
\hline \begin{tabular}{|l|l|l|l|l|l|l|l|l|}
1 & 2 & 3 & 4 & 5 & 6 & 7 & $11 \mid 12$ \\
8 & 9 & 10 & & & & \\
\end{tabular} & \begin{tabular}{|l|l|l|l|l|l|l|}
1 & 2 & 3 & 4 & 5 & 6 & 7 \\
8 & 9 & 10 & 11 & & & \\
\end{tabular} \\
\hline
\end{tabular}

(d) $b=3$

\begin{tabular}{|l|l|l|l|l|l|l||}
\hline 1 & 2 & 3 & 4 & 5 & 7 \\
\hline 8 & 9 & 10 & 11 & 12 & \\
\hline 6 & & & \\
\hline
\end{tabular}

(g) $b=6$

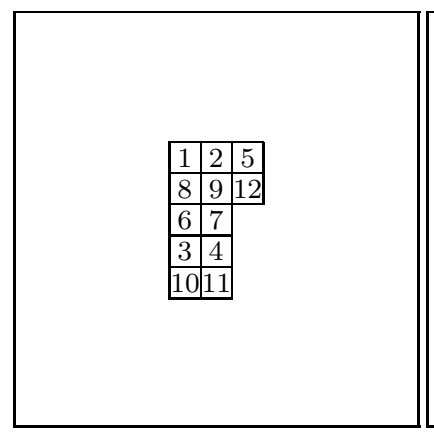

(j) $b=9$ (e) $b=4$

\begin{tabular}{|l|l|l|l|l|}
\hline 1 & 2 & 3 & 4 & 5 \\
\hline 8 & 9 & 10 & 11 & 12 \\
\hline 6 & 7 & & \\
\hline
\end{tabular}

(h) $b=b_{2}=7$

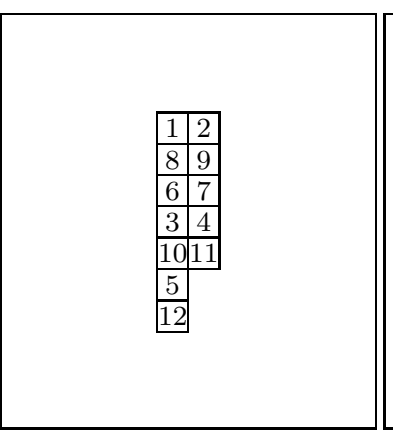

(k) $b=b_{3}=10$ \begin{tabular}{|l|l|l|l|l|l|}
\hline 1 & 2 & 3 & 4 & 5 & 6 \\
\hline 8 & 9 & & & & \\
\hline
\end{tabular} \begin{tabular}{|l|l|l|l|l|}
\hline 8 & 9 & 10 & 11 & 12 \\
\hline
\end{tabular}

(f) $b=b_{1}=5$

\begin{tabular}{|l|l|l|l|l|}
\hline 1 & 2 & 4 & 5 \\
\hline 8 & 9 & 11 & 12 \\
\hline 6 & 7 & \\
\cline { 1 - 1 } 3 & \\
\hline 10 & \\
\hline
\end{tabular}

(i) $b=8$

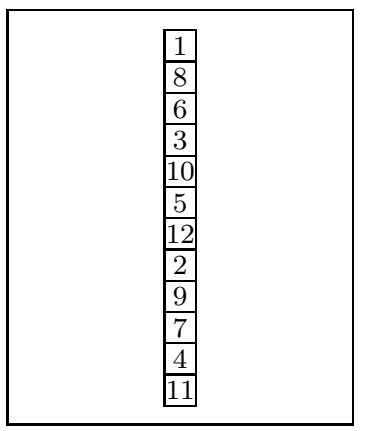

(l) $b=b_{4}=11$

FiguRE 11. Numbered Ferrer diagrams associated to different inclusions of consecutive square-root branch points in the circle $\Xi$, for $p=5$ and $q=12$. In each caption, the number of included branch points $b$ is indicated. The numeration is explained in the text.

In the next subsection, we present a useful link between these two-steps-recursive relations, and the continuedfraction expansion of the number $\frac{1}{1-\mu}$.

4.2. Connection with continued fractions. Let us recall some basic notions about simple continued fractions. Let $x$ be a non-negative real number. We associate to the real number $x$ an integer sequence $\left\{a_{k}\right\}$ such that:

$$
x=a_{0}+\frac{1}{a_{1}+\frac{1}{a_{2}+\frac{1}{a_{3}+\ldots}}} .
$$

We say that $\left\langle a_{0}, a_{1}, a_{2}, \ldots\right\rangle$ is the simple continued fraction of $x$ with $a_{k}$ positive integers $\forall k>0$ (as usual, for the sake of simplicity, we avoid repeating the adjective simple when referring to simple continued fractions). The elements $a_{k}$ of the continued fraction expansion are called partial quotients.

The integer sequence $\left\{a_{k}\right\}$ is finite if and only if the corresponding number $x$ is a rational number. The number of elements in the sequence is called the length of the continued fraction.

The partial quotients can be recursively calculated introducing the auxiliary sequence of the remainders $r_{k}$ :

$$
\left\{\begin{array}{l}
r_{k}=\frac{1}{r_{k-1}\left\lfloor r_{k-1}\right\rfloor}, \quad r_{0}=x, \\
a_{k}=\left\lfloor r_{k}\right\rfloor
\end{array}\right.
$$

We trust no reader will be confused by the similarity of this standard notation for the remainders $r_{k}$ with the radius $r_{b}$, see (22C). The rational number $c_{n} \equiv c_{n}(x)$ obtained truncating the continued fraction of $x$ at 
the $n$-th term is called the $n$-th convergent of the continued fraction:

$$
c_{n}=\frac{P_{n}}{Q_{n}}=\left\langle a_{0}, a_{1}, \ldots, a_{n}\right\rangle=a_{0}+\frac{1}{a_{1}+\frac{1}{\ldots+\frac{1}{a_{n}}}} .
$$

The numerator $P_{n}$ and the denominator $Q_{n}$ of the $n$-th convergent $c_{n}$ satisfy the following second-order recurrence relations:

$$
\begin{array}{lll}
P_{n}=a_{n} P_{n-1}+P_{n-2}, & P_{-2}=0, & P_{-1}=1 ; \\
Q_{n}=a_{n} Q_{n-1}+Q_{n-2}, & Q_{-2}=1, & Q_{-1}=0 .
\end{array}
$$

Now we are ready to rephrase the quantities introduced in the previous subsection in the language of continued fractions. First of all, let us eliminate from the $q$-sequence the dependence on the $p$-sequence in formula (27a):

$$
q_{n}=q_{n-2} \operatorname{MOD}\left(q_{n-1}\right), \quad q_{0}=q, \quad q_{1}=q-p .
$$

We can also write this formula as follows:

$$
\frac{q_{n}}{q_{n-1}}=\frac{q_{n-2}}{q_{n-1}}-\left\lfloor\frac{q_{n-2}}{q_{n-1}}\right\rfloor, \quad q_{0}=q, q_{1}=q-p,
$$

where we used the fact that $\frac{x-[x \operatorname{MOD}(y)]}{y}=\left\lfloor\frac{x}{y}\right\rfloor$ for all $x, y \in \mathbb{N}^{+}$. Now, let us set

$$
r_{k}=\frac{q_{k}}{q_{k+1}} \text { with } 0 \leq k \leq \bar{k}
$$

Using (36), we recover formula (32) for the quantity $r_{k}$,

$$
r_{k}=\frac{1}{r_{k-1}-\left\lfloor r_{k-1}\right\rfloor} \text { with } r_{0}=\frac{q_{0}}{q_{1}}=\frac{q}{q-p}=\frac{1}{1-\mu} .
$$

The $q$-sequence, namely the number of columns in the Ferrer diagrams, can be obtained by inverting formula (37),

$$
q_{k}=\frac{q_{k-1}}{r_{k-1}}=q\left(\prod_{j=0}^{k-1} \frac{1}{r_{j}}\right), \quad q_{0}=q, r_{0}=\frac{1}{1-\mu}, \quad 0 \leq k \leq \bar{k}
$$

where the $r_{k}$ are the terms of the sequence of the remainders (see(38)) of the continued fraction expansion of the number $\frac{1}{1-\mu}$, with partial quotients

$$
a_{k}=\left\lfloor r_{k}\right\rfloor=\left\lfloor\frac{q_{k}}{p_{k}}\right\rfloor
$$

and length $\bar{k}$. The $p$-sequence can be calculated by using the relation:

$$
p_{k}=q_{k+1}, \quad 0 \leq k \leq \bar{k} .
$$

We can moreover provide a convenient reformulation yielding the lengths of the columns in the Ferrer diagrams (30), $T_{k}^{(1)}$ and $T_{k}^{(2)}$. Indeed, by subtracting the two recursion relations (30), one gets

$$
T_{k+1}^{(1)}=T_{k+1}^{(2)}+T_{k}^{(2)},
$$

and the insertion of this last formula in the second of the (30) yields, via (40), a 3-terms linear recursion for $T_{k}^{(2)}$ :

$$
T_{k+1}^{(2)}=a_{k} T_{k}^{(2)}+T_{k-1}^{(2)}
$$

A comparison of this last recursion relation with (34), and an analogous comparison of the starting conditions, yield the relation

$$
T_{k}^{(2)}=P_{k-1}
$$

where $P_{k}$ is the numerator of the $k$-th convergent of the continued fraction expansion of $\frac{1}{1-\mu}$. Then, (42) and (44) imply

$$
T_{k}^{(1)}=P_{k-1}+P_{k-2}
$$


Now all the quantities involved in the description of the Ferrer diagrams (i.e. the numbers of columns and boxes in each column) are expressed in terms of the continued fraction expansion of the (rational) number $\frac{1}{1-\mu}$.

We conclude this section by noting a more convenient way of writing the fundamental $b$-sequence (28a). Indeed, by combining (37), (38) and (40), one gets:

$$
q_{k}=q_{k-2}-a_{k-2} q_{k-1}, \quad q_{0}=q, \quad q_{1}=q-p,
$$

and by comparing this last relation with the recursion relations (34) via the following ansatz:

$$
q_{k}=(-1)^{k}\left(\alpha P_{k-2}+\beta Q_{k-2}\right), k \geq 0,
$$

one immediately gets

$$
\alpha=(p-q), \quad \beta=q ;
$$

the insertion of (46b) and (46c) in (28b) entails

$$
b_{k}=q-q_{k}=q-(-1)^{k}\left[(p-q) P_{k-2}+q Q_{k-2}\right], \quad k \geq 0 .
$$

\section{Determination OF THE PERIOD}

5.1. The period formula for $\mu \in \mathbb{Q}$. Once the structure of the Riemann surface has been achieved, our next step is to provide an explicit formula to predict the period of each of the $q$ roots of the algebraic equation (21).

The variable $\xi$ moves along the circle $\Xi$ which includes $b$ consecutive square-root branch points on the $\xi$-plane, starting from $\xi_{\mathrm{b}}^{(1)}$. Now we introduce a numbering of the boxes of the Ferrer diagrams, so that, if one fixes a single column, all the roots labeled with the numbers appearing in the chosen column have the same period, equal to the length of the column. For instance, look at Figure 11(i) when $b=8$. We interpret this picture, inferring that the roots $w_{1}, w_{3}, w_{6}, w_{8}$ and $w_{10}$ have period 5; the roots $w_{2}$, $w_{7}$ and $w_{9}$ have period 3 ; the roots $w_{4}, w_{5}, w_{11}$ and $w_{12}$ have period 2 . This is exactly what you obtain by comparing with Figure 10(i).

For fixed $p$ and $q$, if we enumerate the boxes of $F(0)$, starting from the left, with the first consecutive $q$ natural numbers $(1,2, \ldots, q)$ and if we apply recursively the bumping rule, then we obtain the correct enumeration for each $F(b)$ with $b>0$.

Let us introduce the number $l_{h}(s)$, defined recursively as follows:

$$
l_{h}(s)=l_{h-1}(s) \text { MÕD }\left(p_{h-1}\right), \quad l_{0}(s)=s,
$$

for all $s=1,2, \ldots, q$ and for all $h=1,2, \ldots, \bar{k}$. This number $l_{h}(s)$ gives the position of the column where the box with the number $s$ lies in the $h$-level Ferrer diagram. Once we know this information for a $h$-level Ferrer diagram, we can extend the same information for a generic Ferrer diagram.

Let us suppose that there are $b$ branch points included in the circle $\Xi$, with $b_{h} \leq b<b_{h+1}$. We are interested in the period of $w_{s}$. Then two cases are possible.

Case 1: If $l_{h}(s)>b-b_{h}+p_{h}$ then $T_{h}^{(2)}$ is the length of the column where the box with number $s$ lies; this column was not moved from the position it occupied when exactly $b_{h}$ branch points were included in the circle $\Xi$.

Case 2: If $l_{h}(s) \leq b-b_{h}+p_{h}$ then we must consider two subcases: if $l_{h}(s) \leq p_{h}$, then the length of the column in $F\left(b_{h}\right)$, containing the box $s$ and lying at the position $l_{h}(s)$, is $T_{h}^{(1)} ;$ if $l_{h}(s)>p_{h}$, then the length of the column in $F\left(b_{h}\right)$, containing the box $s$ and lying at the position $l_{h}(s)$, is $T_{h}^{(2)}$. In both cases, in order to obtain $F(b)$ starting from $F\left(b_{h}\right)$, the bumping rule predicts that, if the circle $\Xi$ includes other $b-b_{h}$ branch points, then some of the last $\left(q_{h}-p_{h}\right)$ columns in $F\left(b_{h}\right)$, with length $T_{h}^{(2)}$, are moved under the column located at the position $l_{h}(s)$ MÕD $\left(p_{h}\right)$. If $l_{h}(s) \leq p_{h}$, then $l_{h}(s)$ MÕD $\left(p_{h}\right)=l_{h}(s)$ and the column at the position $l_{h}(s)$ in $F(b)$ contains the box $s$. If $l_{h}(s)>p_{h}$, then the column in $F\left(b_{h}\right)$, at the position $l_{h}(s)$ and containing the box $s$, is moved under the column in $F(b)$ lying at the position $l_{h}(s)$ MÕD $\left(p_{h}\right)$. Thus, in both cases, the column in $F(b)$ at the position $l_{h}(s)$ MÕD $\left(p_{h}\right)$ contains the box $s$. On the other hand, the number of moved columns depends on whether $l_{h}(s)$ MÕD $\left(p_{h}\right)$ is smaller than $\left(b-b_{h}\right)$ MÕD $\left(p_{h}\right)$ or not. If $l_{h}(s)$ MÕD $\left(p_{h}\right)<\left(b-b_{h}\right)$ MÕD $\left(p_{h}\right)$ then the number of columns of length $T_{h}^{(2)}$, moved under the 
column at the position $l_{h}(s)$ MÕD $\left(p_{h}\right)$, is $\left(\left\lfloor\frac{b-b_{h}-1}{p_{h}}\right\rfloor\right)$. If $l_{h}(s)$ MÕD $\left(p_{h}\right) \geq\left(b-b_{h}\right)$ MÕD $\left(p_{h}\right)$ then one needs to move one more column of length $T_{h}^{(2)}$, so their total number is $\left(\left\lfloor\frac{b-b_{h}-1}{p_{h}}\right\rfloor+1\right)$.

From the above considerations, we finally infer the following

Theorem 1. Assume $0<\mu<1$ and $\mu \in \mathbb{Q}$. Let the roots of the algebraic equation (21) be labeled as described in Subsection 3.3. Let $T(s, b)$ be the period of $w_{s}$, the $s$-th root of the algebraic equation (21) when $\xi$ moves along a closed path on the $\xi$-plane, including $b$ consecutive adjacent branch points starting from the branch point $\xi_{\mathrm{b}}^{(1)}$. Let $h$ be the integer such that $0 \leq b_{h} \leq b<b_{h+1} \leq q-1$ and $l_{h}(s)$ the symbol 48). Let $T_{h}^{(1)}$ and $T_{h}^{(2)}$ be the quantities described by the recursions (30). Then we have the following period formula, for all $b<q$ :

$$
T(s, b)= \begin{cases}T_{h}^{(1)}+\left(\left\lfloor\frac{b-b_{h}-1}{p_{h}}\right\rfloor\right) T_{h}^{(2)} & \text { if } l_{h}(s) \leq b-b_{h}+p_{h} \text { and } \\ \left(b-b_{h}\right) \text { MÕD }\left(p_{h}\right)<l_{h}(s) \mathrm{MÕD}\left(p_{h}\right) \\ T_{h}^{(1)}+\left(\left\lfloor\frac{b-b_{h}-1}{p_{h}}\right\rfloor+1\right) T_{h}^{(2)} & \text { if } l_{h}(s) \leq b-b_{h}+p_{h} \text { and } \\ & \left(b-b_{h}\right) \mathrm{MÕD}\left(p_{h}\right) \geq l_{h}(s) \mathrm{MÕD}\left(p_{h}\right) \\ T_{h}^{(2)} & \text { if } l_{h}(s)>b-b_{h}+p_{h} .\end{cases}
$$

If $b=q$ we have

$$
T(s, q)= \begin{cases}q-p & \text { if } 1 \leq s \leq q-p \\ p & \text { if } q-p+1 \leq s \leq q .\end{cases}
$$

Via the connection with continued fractions, as shown in Subsection 4.2. we can reformulate 49a]) as follows:

$$
T(s, b)= \begin{cases}P_{h-2}+\left(\left\lfloor\frac{b-b_{h}-1}{q-b_{h+1}}\right\rfloor+1\right) P_{h-1} & \text { if } l_{h}(s) \leq b+q-\left(b_{h}+b_{h+1}\right) \text { and } \\ & \left(b-b_{h}\right) \text { MÕD }\left(q-b_{h+1}\right)<l_{h}(s) \text { MÕD }\left(q-b_{h+1}\right) \\ P_{h-2}+\left(\left\lfloor\frac{b-b_{h}-1}{q-b_{h+1}}\right\rfloor+2\right) P_{h-1} & \text { if } l_{h}(s) \leq b+q-\left(b_{h}+b_{h+1}\right) \text { and } \\ & \left(b-b_{h}\right) \text { MÕD }\left(q-b_{h+1}\right) \geq l_{h}(s) \text { MÕD }\left(q-b_{h+1}\right) \\ & \text { if } l_{h}(s)>b+q-\left(b_{h}+b_{h+1}\right),\end{cases}
$$

where $P_{k}$ is the numerator of the $k$-th convergent of the continued fraction expansion of $\frac{1}{1-\mu}$.

For any inclusion of consecutive branch points, the final formula (50) indicates that the roots of the algebraic equation (21) can feature only three possible periods (see Table 1). Note that the sum of the first and third expressions in the right hand side of this formula, (50), always gives the second expression. Moreover, if $b$ takes the special values $b=b_{h}+n\left(q-b_{h+1}\right), 0 \leq n \leq a_{h}-1, n \in \mathbb{N}$, where $a_{h}$ is the $h$-th partial quotient appearing in the continued fraction expansion of $\frac{1}{1-\mu}$, then the roots of (21) can feature only two possible periods: indeed, in these particular cases, the first condition in (50) fails for all $s$ since $\left(b-b_{h}\right)$ MÕD $\left(q-b_{h+1}\right)=q-b_{h+1}$ and $1 \leq l_{h}(s)$ MÕD $\left(q-b_{h+1}\right) \leq q-b_{h+1}$.

Summarizing, given the initial data $z_{i}(0), i=1,2,3$, and the coupling constants $f, g$ (such that $\mu \in \mathbb{Q}$ ) we can now predict the period of the function $\breve{w}(t)$ by the following prescription:

(1) Draw the circles $\Xi$ and $B$ defined by (14b) and (10b).

(2) Identify the label of the physical root $\breve{s}$ following the prescription given in Section 3.4 .

(3) Calculate the number of branch points $b$ included in the circle $\Xi$.

(4) Develop in continued fraction the ratio $\frac{1}{1-\mu}$ and build the sequence $b_{k}$ defined by (47). Identify the element $h$ of the sequence such that $b_{h} \leq b<b_{h+1}$. Calculate $l_{h}(\check{s})$ using (48).

(5) The period $T(\check{s}, b)$ of $\check{w}(t)$ is given by Theorem 1 .

The understanding of the topology of the Riemann surface $\Gamma$ for $\mu \in \mathbb{Q}$ allows one to associate the evolution in time of the physical problem (1) to the symbolic dynamics given by the sequence of the labels of the visited sheets of $\Gamma$. In other words, one can describe the time-T map obtained by sampling the solution at every 


\begin{tabular}{||c||c|c|c|c|c|c|c|c|c|c|c|c||}
\hline \hline$s=$ & 1 & 2 & 3 & 4 & 5 & 6 & 7 & 8 & 9 & 10 & 11 & 12 \\
\hline \hline$b=0$ & 1 & 1 & 1 & 1 & 1 & 1 & 1 & 1 & 1 & 1 & 1 & 1 \\
$b=1$ & 2 & 1 & 1 & 1 & 1 & 1 & 1 & 2 & 1 & 1 & 1 & 1 \\
$b=2$ & 2 & 2 & 1 & 1 & 1 & 1 & 1 & 2 & 2 & 1 & 1 & 1 \\
$b=3$ & 2 & 2 & 2 & 1 & 1 & 1 & 1 & 2 & 2 & 2 & 1 & 1 \\
$b=4$ & 2 & 2 & 2 & 2 & 1 & 1 & 1 & 2 & 2 & 2 & 2 & 1 \\
$b=5$ & 2 & 2 & 2 & 2 & 2 & 1 & 1 & 2 & 2 & 2 & 2 & 2 \\
$b=6$ & 3 & 2 & 2 & 2 & 2 & 3 & 1 & 3 & 2 & 2 & 2 & 2 \\
$b=7$ & 3 & 3 & 2 & 2 & 2 & 3 & 3 & 3 & 3 & 2 & 2 & 2 \\
$b=8$ & 5 & 3 & 5 & 2 & 2 & 5 & 3 & 5 & 3 & 5 & 2 & 2 \\
$b=9$ & 5 & 5 & 5 & 5 & 2 & 5 & 5 & 5 & 5 & 5 & 5 & 2 \\
$b=10$ & 7 & 5 & 7 & 5 & 7 & 7 & 5 & 7 & 5 & 7 & 5 & 7 \\
$b=11$ & 12 & 12 & 12 & 12 & 12 & 12 & 12 & 12 & 12 & 12 & 12 & 12 \\
$b=12$ & 7 & 7 & 7 & 7 & 7 & 7 & 7 & 5 & 5 & 5 & 5 & 5 \\
\hline \hline
\end{tabular}

TABLE 1 . Values of $T(s, b)$ when $p=5$ and $q=12$.

time interval $T$, as a sequence of natural numbers, i.e. the sequence of numbers labeling the visited sheets, after each round trip as $\xi$ travels on the Riemann surface.

5.2. The period formula for $\mu \notin \mathbb{Q}$. We treat the case in which $\mu$ is an irrational number as a limit of the case in which $\mu$ is a rational number. If $\mu=p / q$ gets close to being irrational, the number $q$ - that corresponds to the number of roots of equation (21) as well as the number of branch points of $w(\xi)$ in the complex $\xi$-plane - approaches infinity. So we must analyze all the previous formulae in the limit:

$$
p, q \rightarrow \infty \text { with } 0<\mu=\frac{p}{q}<1,
$$

namely in the limit in which the integers $p$ and $q$ diverge so that their ratio tends to the irrational number $\mu$.

If $\mu$ is an irrational number, it follows that the Riemann surface $\Gamma$ associated to (11) becomes an $\infty$-sheeted covering of the complex $\xi$-plane. In the limit (51), the continued fraction expansion of $\frac{1}{1-\mu}$ has length $\bar{k}$ that tends to infinity: $\bar{k} \rightarrow \infty$. The first step is to normalize the $q$-sequence (35), introducing the new sequence $\left\{\rho_{k}\right\} \sim\left\{\frac{q_{k}}{q}\right\}:$

$$
\rho_{k}=\frac{\rho_{k-1}}{r_{k-1}}, \quad \rho_{0}=1, \quad \rho_{1}=1-\mu,
$$

where $r_{k}$ is the sequence of the (irrational) remainders of the continued fraction of $\frac{1}{1-\mu}$ (see (38)). In the limit (51), the $\rho$-sequence becomes a strictly decreasing sequence of irrational numbers such that $\rho_{k} \rightarrow 0$ as $k \rightarrow \infty$.

The next step is to obtain the proper continuous variable which replaces the discrete index $b$. Let $B$ be the circle on which the square-root branch points lie and $\Xi$ the circle on which the $\xi$ variable travels periodically, see (10b). We define the variable $\nu$, which is 0 if $\Xi$ does not contain any arc of $B$ and 1 if $\Xi$ does contain the whole circle $B$. If $B$ and $\Xi$ intersect, select the arc of the $B$ circle which is contained inside the evolutionary circle $\Xi$ and denote by $\nu$ the corresponding angle $\phi$, normalized by $2 \pi$ (see Figure 12). Since

$$
\nu=\frac{\phi}{2 \pi}=\frac{b}{q}
$$

this is a convenient continuous variable appropriate to replace $b$ in the case $\mu$ is an irrational number.

We then normalize the fundamental $b$-sequence (47), replacing it by the new sequence $\left\{\nu_{k}\right\} \sim\left\{\frac{b_{k}}{q}\right\}$ :

$$
\nu_{k}=1-\rho_{k}, \quad \nu_{0}=0, \quad k \in \mathbb{N} ;
$$

in the limit (51), we have that $\nu_{k} \rightarrow 1$ as $k \rightarrow \infty$. It is also convenient to reformulate $\nu_{k}$ using the language of continued fractions. From (47), in the limit (51) we have:

$$
\nu_{k}=1-(-1)^{k}\left[(\mu-1) P_{k-2}+Q_{k-2}\right], \quad k \in \mathbb{N} .
$$

We are finally ready to enunciate the period formula when $\mu$ is an irrational number. 


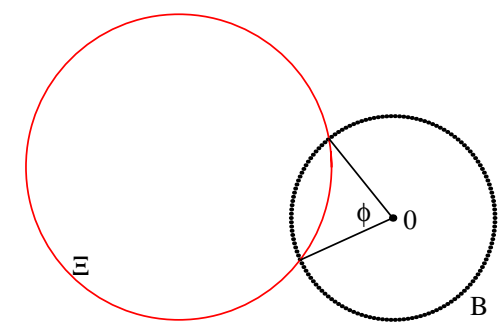

FIgURE 12. The definition of the angle $\phi$

Theorem 2. Let $0<\mu<1$ and $\mu \notin \mathbb{Q}$. Let $T(\nu)$ be the period of one of the roots of the algebraic equation (11) when $\xi$ travels on the circle $\Xi$ in the complex $\xi$-plane, intersecting the square-root branch points circle $B$ in such a way that $0 \leq \nu<1$ (see (53)). Let $h$ be the integer such that $0 \leq \nu_{h} \leq \nu<\nu_{h+1}<1$. Let $P_{k}$ be the numerator of the $k$-th convergent of the continued fraction expansion of $\frac{1}{1-\mu}$ (see (34)). If $\nu=0$, then $T(\nu)=1$. Otherwise, $T(\nu)$ takes one of the following three values:

$$
T(\nu)=\left\{\begin{array}{l}
P_{h-2}+\left(\left\lfloor\frac{\nu-\nu_{h}}{1-\nu_{h+1}}\right\rfloor+1\right) P_{h-1}, \\
P_{h-2}+\left(\left\lfloor\frac{\nu-\nu_{h}}{1-\nu_{h+1}}\right\rfloor+2\right) P_{h-1}, \\
P_{h-1} .
\end{array}\right.
$$

If $\nu=1$, namely if the evolutionary circle $\Xi$ contains entirely the square-root branch points circle $B$, then the dynamics becomes again simple, because the evolutionary curve effectively surrounds only the irrational branch point at infinity. In this latter case, the time evolution of the generic root of (11) is quasi-periodic, involving a (nonlinear) superposition of two periodic evolutions with noncongruent periods, 1 and $\frac{1}{\mu}$ or 1 and $\frac{1}{1-\mu}$, as can be easily understood observing the exponents of the asymptotics (15a)-(15c) and (16a)-(16c).

Note that, as in the rational case, the sum of the first and third expressions in the right hand side of (55) always gives the second expression. We remark that in the irrational case there are no labels for the roots, so we cannot assign the periods to the roots in terms of their labels as we did in the rational case. Therefore, a specific prescription to identify the period of the physical root $\breve{w}(t)$ cannot be given. At best, we can assure that the period is one of the three possibilities specified by (55).

For $\nu=0$, the period $T(0)$ is equal to 1 . Since 1 is the accumulation point of the sequence $\left\{\nu_{k}\right\}$, if $\nu$ is instead close to 1 (i.e. if the evolutionary circle $\Xi$ contains almost completely the branch points circle $B)$, a small change in the time trajectory results in a drastic change of the observed periods. In fact, $T(\nu)$ approaches infinity as $\nu \rightarrow 1$ and the values of $T(\nu)$ depend on the partial quotients $a_{k}$ of the continued fraction expansion of $\frac{1}{1-\mu}$; such partial quotients are well-known to be chaotic and unpredictable in their sequence for a generic irrational number (for almost all the irrational numbers, except for the quadratic irrationals that feature periodic continued fraction expansions).

In the next subsection we treat a remarkable example where it is possible to obtain explicitly the asymptotic behavior of $T(\nu)$ as $\nu \sim 1$.

5.3. A remarkable example. In this subsection we display the special example with the following (conveniently chosen) quadratic irrational value of $\mu$ in the interval $0<\mu<1$ :

$$
\mu=\frac{2}{3+\sqrt{5}}=\frac{1}{1+\varphi}
$$

such that

$$
\frac{1}{1-\mu}=\varphi=\frac{1+\sqrt{5}}{2}
$$

where $\varphi$ is the so-called golden ratio, namely the positive solution of the second degree equation

$$
\varphi^{2}-\varphi-1=0 .
$$


The golden ratio $\varphi$ has the nice property that all the infinite partial quotients (40) appearing in its continued fraction expansion are equal to unity:

$$
\varphi=1+\frac{1}{1+\frac{1}{1+\frac{1}{1+\frac{1}{\cdots}}}}, \quad a_{k}=1 \forall k \geq 0 .
$$

So, in this particular case, the recursion relation (38) becomes:

$$
r_{k}=\frac{1}{r_{k-1}-1} \text { with } r_{0}=\varphi, k \geq 1 .
$$

By combining this last relation with (52) we get:

$$
\rho_{k+1}=\rho_{k-1}-\rho_{k} \text { with } \rho_{0}=1, \rho_{1}=\frac{1}{\varphi}=\varphi-1, k \geq 1
$$

and from this, via (54a),

$$
\nu_{k+1}=\nu_{k-1}-\nu_{k}+1 \text { with } \nu_{0}=0, \nu_{1}=1-\frac{1}{\varphi}, k \geq 1 .
$$

Solving this last relation with respect to $k$, we get

$$
\nu_{k}=1-\varphi^{-k}, k \geq 0 .
$$

Moreover - somewhat remarkably - (57) implies, via (34), the following relation for the numerators of the convergents:

$$
P_{k}=P_{k-1}+P_{k-2}, P_{-2}=0, P_{-1}=1, k \geq 0,
$$

i.e. exactly the recurrence relation for Fibonacci's numbers. Using Binet formula, we have:

$$
P_{k}=\frac{1}{\sqrt{5}}\left[\varphi^{k+2}-(-\varphi)^{-(k+2)}\right], k \geq-2 .
$$

Through (61), we can explicitly invert the inequality $\nu_{k} \leq \nu<\nu_{k+1}$, finding, for a fixed value of $\nu$ in the interval $0<\nu<1$, the integer number $k$ such that $\nu_{k} \leq \nu<\nu_{k+1}$ :

$$
k \equiv k(\nu)= \begin{cases}-\left\lfloor\frac{\log (1-\nu)}{\log (\varphi)}\right\rfloor-1, & \text { if } 0<\nu<1 ; \\ 0, & \text { if } \nu=0\end{cases}
$$

Using (64), we see that the argument of the floor function in (55) must satisfy the inequalities:

$$
0<\frac{\nu-\nu_{k(\nu)}}{1-\nu_{k(\nu)+1}}<\varphi-1<1 ;
$$

so the floor function in (55) always vanishes and the root period $T(\nu)$ has one of the following values:

$$
T(\nu)=\left\{P_{k+1}, P_{k}, P_{k-1}\right\} \quad \text { with } 0 \leq \nu_{k} \leq \nu<\nu_{k+1}<1
$$

namely one of three consecutive Fibonacci's numbers. From (62), via (64), we see that, for $0<\nu<1$,

$$
\frac{1}{\sqrt{5}}\left[\frac{\varphi}{1-\nu}-\frac{1-\nu}{\varphi}\right]<P_{k(\nu)}<\frac{1}{\sqrt{5}}\left[\frac{\varphi}{1-\nu}+\frac{1-\nu}{\varphi}\right] .
$$

From the above relations (67) and (65), and from the period formula (55), we obtain the following lower and upper bounds for the period values in terms of $\nu$ in the interval $0<\nu<1$ :

$$
\frac{\nu(2-\nu)}{\sqrt{5}(1-\nu)} \leq T(\nu)<\frac{7+\sqrt{5}+(\sqrt{5}-3) \nu(2-\nu)}{2 \sqrt{5}(1-\nu)} .
$$

These inequalities entail that the integer $T(\nu)$ diverges proportionally to $(1-\nu)^{-1}$ as $\nu \rightarrow 1$. 


\section{Summary AND CONCLUSIONS}

We have studied the trajectories of the system of three coupled ODEs (11) in the semi-symmetrical case (4) when two coupling constants are equal, and the ratio $\mu$ defined in (5) belongs to the interval $(0,1)$.

For rational values of $\mu$, all orbits are periodic for arbitrary initial data, except for a set of null measure that corresponds to a collision in finite time. Moreover, the system is isochronous: the periodic orbits are neutrally stable and a small perturbation of the initial condition produces another periodic orbit of the same period. In this case, the Cauchy problem can be solved explicitly and the evolution in time of the physical trajectories can be put in correspondence with the symbolic dynamics (a sequence of natural numbers) of the labels of the visited sheets of the associated Riemann surface.

For irrational values of the ratio $\mu$, one must distinguish two cases depending on the relative position of the evolutionary circle $\Xi$, defined by (10b), with respect to the branch-point circle $B$, defined by (14b). If $B$ is entirely contained in $\Xi$, then the solution is quasi-periodic, while if $\Xi$ contains only a part of $B$, then almost all orbits are periodic (again, except for a set of null measure that corresponds to a collision in finite time). The relative position of these two circles depends on the choice of initial data. In fact, initial data can always be found such that the period of the corresponding orbit is as high as desired (although always a multiple of the fundamental period). This situation corresponds to the limiting case between the two cases described above. An explicit instance of this situation has been given for a particularly simple example related to the golden ratio and Fibonacci's numbers.

The main result of this paper is to derive explicit formulae for the period as a function of the initial data and the coupling constants. The only way the period of an orbit can change as the initial data are varied is passing through a singularity (corresponding to a collision between the particles). The dependence of the period on the initial data is shown to depend on the continued fraction expansion of the parameter $\mu$, which for irrational values shows a rich behavior. We would like to emphasize the novelty of the approach developed in this paper: it is not possible to achieve such an explicit description of the dynamics of the system using the qualitative theory of ODEs, which illustrates why the complete description of the Riemann surface is an important goal in the cases where it can be achieved. The description performed in this case involves the use of techniques that range from complex analysis and geometry to graph theory and continued fractions, which shows the novelty of the approach.

One of the important lessons from the results in this paper in relation to the ideas of Kruskal, Bountis, Tabor and their collaborators mentioned in the Introduction is the following: the fact that the Riemann surface associated to the solution of the problem is infinitely sheeted is not enough for a system to produce chaotic behaviour, although it can be a source of unpredictable long term behaviour. This is illustrated by the behaviour of the semi-symmetric three-body problem with an irrational value of $\mu$ (i.e. generic values of the coupling constants). In this case, we have shown that the Riemann surface is infinitely-sheeted and it contains an infinite number of branch points whose position depends on the initial data and whose projection on the complex plane fills densely a circle $B$. Furthermore, we have proved that all solutions are either periodic, quasi-periodic or lead to a collision in finite time. Despite this fact, even in the periodic case there is a source of unpredictability associated to the fact that it is not possible to identify the root associated to the physical problem among the infinity of roots of (11). Moreover, two trajectories originating from nearby initial data will separate at some future time as they will travel to different sheets on the Riemann surface, corresponding to the fact that the collision manifolds (locus of initial data in phase space that lead to a collision in finite time) fill densely an open set of phase space. On the other hand, in the $\mu$-rational case, not only can the period be predicted by an explicit formula, but the complete motion can be described by the sequence of sheets in the Riemann surface that are visited during the motion.

The description of the remaining case $\mu>1$ can be tackled with similar techniques to the ones developed in this paper. However, we have decided to postpone this analysis to a further publication, where the sensitive dependence on the initial conditions will be discussed. An interesting further step in this program would be to understand how sensitive dependence or classical indicators of chaos such as Lyapunov exponents are related to properties of the Riemann surface.

Acknowledgements. It is a pleasure to acknowledge illuminating discussions with Carl Bender, Boris Dubrovin, Yuri Fedorov, Jean-Pierre Françoise, Peter Grinevich and François Leyvraz. The research of DGU was supported in part by MICINN-FEDER grant MTM2009-06973 and CUR-DIUE grant 2009SGR859 and 
he would like to thank the financial support received from the Università di Roma "La Sapienza" under the

Accordo Bilaterale with Universidad Complutense de Madrid.

\section{REFERENCES}

[1] P. Painlevé. Mémoire sue les équations différentielles du premier ordre, Annales Scientifiques de l'ENS 8: 9-58 (1891).

[2] S. Kowalewskaya. Sur une propriété du système d'équations différentielles qui définit la rotation d'un corps solide autour d'un point fixe, Acta Mathematica 14:81-93 (1890).

[3] A. Ramani, B. Grammaticos and T. Bountis. The Painlevé property and singularity analysis of integrable and nonintegrable systems, Phys. Rep. 180: 159-245 (1989).

[4] D. Balwin and W. Hereman. Symbolic software for the Painlevé test of nonlinear ordinary and partial differential equations, J. Nonlin. Math. Phys. 13: 90-110 (2006).

[5] T. Bountis, H. Segur and F. Vivaldi. Integrable Hamiltonian systems and the Painlevé property, Phys. Rev. A 25: 1257-1264 (1982).

[6] Y. F. Chang, M. Tabor, and J. Weiss. Analytic structure of the Hénon-Heiles Hamiltonian in integrable and nonintegrable regimes, J. Math. Phys. 23: 531-538 (1982).

[7] M. D. Kruskal and P. A. Clarkson. The Painlevé-Kowalewski and poly-Painlevé tests for integrability, Studies Appl. Math. 86: 87-165 (1992).

[8] M. D. Kruskal, A. Ramani and B. Grammaticos. Singularity analysis and its relation to complete, partial and nonintegrability, in Partially integrable evolution equations in physics (Les Houches, 1989), NATO Adv. Sci. Inst. Ser. C Math. Phys. Sci. 310: 321-372, Kluwer Acad. Publ., Dordrecht, 1990.

[9] M. Tabor and J. Weiss. Analytic structure of the Lorenz system, Phys. Rev. A 24: 2157-2167 (1981).

[10] Y. F. Chang, J. M. Greene, M. Tabor and J. Weiss. The analytic structure of dynamical systems and self-similar natural boundaries, Physica D 8: 183-207 (1983).

[11] G. Levine and M. Tabor. Integrating the nonintegrable: analytic structure of the Lorenz system revisited, Physica D 33: 189-210 (1988).

[12] M. Tabor. Chaos and integrability in nonlinear dynamics: An introduction, Wiley-Interscience, New York, 1989.

[13] Y. F. Chang and G. Corliss. Ratio-like and recurrence relation tests for convergence of series, J. Inst. Math. Appl. 25: 349-359 (1980).

[14] T. Bountis. Investigating non-integrability and chaos in complex time, Physica D 86: 256-267 (1995).

[15] T. Bountis, L. Drossos and I. C. Percival. Nonintegrable systems with algebraic singularities in complex time, J. Phys. A 24: 3217-3236 (1991).

[16] A. S. Fokas and T. Bountis. Order and the ubiquitous occurrence of chaos, Physica A 228: 236-244 (1996).

[17] A. G. Anderson, C. M. Bender and U. I. Morone. Periodic orbits for classical particles having complex energy, arXiv:1102.4822v1 (2001).

[18] C. M. Bender, D. D. Holm and D. W. Hook. Complex trajectories of a simple pendulum, J. Phys. A 40: F81-F89 (2007).

[19] C. M. Bender, D. W. Hook, P. N. Meisinger and Q. H. Wang. Probability density in the complex plane, Ann. Physics 325: 2332-2362 (2010).

[20] C. M. Bender, D. W. Hook and K. S. Kooner. Classical particle in a complex elliptic potential, J. Phys. A 43: 165201 (2010).

[21] C. M. Bender, J. Feinberg, D. W. Hook and D. J. Weir. Chaotic systems in complex phase space, Pramana J. Phys. 73: 453-470 (2009).

[22] F. Calogero, D. Gomez-Ullate, P. M. Santini and M. Sommacal. On the transition from regular to irregular motions, explained as travel on Riemann surfaces, J. Phys. A 38: 8873-8896 (2005).

[23] F. Calogero, D. Gomez-Ullate, P. M. Santini and M. Sommacal. Towards a theory of chaos explained as travel on Riemann surfaces, J. Phys. A 42: 015205 (2009).

[24] F. Calogero. A class of integrable Hamiltonian systems whose solutions are (perhaps) all completely periodic, J. Math. Phys. 38: 5711-5719 (1997).

[25] F. Calogero. Classical many-body problems amenable to exact treatments, Lecture Notes in Physics Monograph m 66, Springer, Berlin, 2001.

[26] F. Calogero. Isochronous systems, Oxford University Press, Oxford, 2008.

[27] F. Calogero and M. Sommacal. Periodic solutions of a system of complex ODEs. II. Higher periods, J. Nonlin. Math. Phys. 9: $1-33(2002)$.

[28] F. Calogero and J.-P. Françoise. Periodic motions galore: how to modify nonlinear evolution equations so that they feature a lot of periodic solutions, J. Nonlin. Math. Phys. 9: 99-125 (2002).

[29] F. Calogero, J.-P. Françoise and M. Sommacal. Periodic solutions of a many-rotator problem in the plane. II. Analysis of various motions, J. Nonlin. Math. Phys. 10: 157-214 (2003).

[30] D. Gomez-Ullate and M. Sommacal. Periods of the Goldfish many-body problem, J. Nonlin. Math. Phys. 12: $351-362$ (2005).

[31] Yu. Fedorov and D. Gomez-Ullate. A class of dynamical systems whose solutions travel on the Riemann surface of an hyperelliptic function, Physica D 227: 120-134 (2007).

[32] P. Grinevich and P. M. Santini. Newtonian dynamics in the plane corresponding to straight and cyclic motions on the hyperelliptic curve $\mu^{2}=v^{n}-1, n \in \mathbb{Z}$ : ergodicity, isochrony, periodicity and fractals, Physica D 232: 22-32 (2007).

[33] M. Sommacal. The transition from regular to irregular motions, explained as travel on Riemann surfaces, PhD Thesis, SISSA-ISAS, Trieste, 2005. 
Departamento de Física Teórica II, Universidad Complutense, Madrid, Spain.

E-mail address, D. Gomez-Ullate: david.gomez-ullate@fis.ucm.es

Dipartimento di Fisica, Università di Roma "La Sapienza", Roma, Italy.

Istituto Nazionale di Fisica Nucleare, Sezione di Roma, Italy.

E-mail address, P. M. Santini: paolo.santini@roma1.infn.it

Institut des Hautes Etudes Scientifiques, Bures-Sur-Yvette, France.

E-mail address, M. Sommacal: sommacal@ihes.fr

Istituto Nazionale di Fisica Nucleare, Sezione di Roma, Italy.

E-mail address, F. Calogero: francesco.calogero@roma1.infn.it 